\title{
ON THE COMBINED EFFECT OF BOUNDARY APPROXIMATION AND NUMERICAL INTEGRATION ON MIXED FINITE ELEMENT SOLUTION OF 4TH ORDER ELLIPTIC PROBLEMS WITH VARIABLE COEFFICIENTS
}

\author{
Pulin K. Bhattacharyya ${ }^{1}$ and Neela NataraJ ${ }^{2}$
}

\begin{abstract}
Error estimates for the mixed finite element solution of 4th order elliptic problems with variable coefficients, which, in the particular case of aniso-/ortho-/isotropic plate bending problems, gives a direct, simultaneous approximation to bending moment tensor field $\Psi=\left(\psi_{i j}\right)_{1 \leq i, j \leq 2}$ and displacement field ' $u$ ', have been developed considering the combined effect of boundary approximation and numerical integration.
\end{abstract}

AMS Subject Classification. 35J40, 65N30.

Received: January 6, 1998. Revised: October 23, 1998.

\section{INTRODUCTION}

In [5] a new mixed finite element method for 4th order elliptic partial differential equations with variable/constant coefficients defined in convex polygonal domain, from which the mixed method scheme of HellanHermann-Miyoshi $[15,22,23,28]$ for the biharmonic problem in convex polygonal domain can be retrieved as a particular case with a proper choice of coefficients $a_{i j k l}$ of the equation [see (2.2)], was developed with all details of mathematical analysis of convergence. This mixed finite element method found its application in the mixed method analysis of shell problems in [31] and also specific mention in [33]. But for the same isotropic plate bending problem, the mixed method scheme of [5] and that of Hellan-Hermann-Miyoshi are different. Error estimates of order $O\left(h^{m-1}\right)$ have been obtained in [5] under the assumption that an exact integration of the integrals of the bilinear forms is possible, the domain being a convex polygonal one (i.e. no approximation of the boundary is necessary), the convexity of the polygonal domain (in all papers) being a requirement for the regularity $[21,24]$ of the solution on which the proof of the existence of solution of the continuous mixed variational problem and error estimates are based. But in many practical situations both approximation of the curved boundary of the convex domain by a polygonal one or some other suitable curved boundary and numerical integration for the evaluation of bilinear forms are to be performed. In such situations an estimate for the combined effect of the numerical integration and approximation of the curved boundary of the convex domain on the mixed finite element solution of the problem is essential. Such estimates for classical finite element methods of solution of second order problems have been obtained in [17,19,32,35,36,38-40], and of fourth

Keywords and phrases. Mixed finite element method, fourth order equations, convergence analysis.

${ }^{1}$ Professor, Department of Mathematics, Indian Institute of Technology, New Delhi 110016, India.

Present Address: Honorary Visiting Professor, School of Computer and Systems Sciences, Jawaharlal Nehru University, New Delhi 110067, India. e-mail: pulinkum@hotmail.com

${ }^{2}$ Research Scholar, Department of Mathematics, Indian Institute of Technology, New Delhi 110016, India.

e-mail: neela@maths.iitd.ernet.in

(C) EDP Sciences, SMAI 1999 
order problems in $[8,27]$, but to our knowledge such results for mixed finite element methods for fourth order problems are conspicuous by their absence in published research literature. Moreover, construction of estimates for these combined effects on mixed method solution for fourth order problems is associated with mathematical difficulties. The present paper contains new, original results in this direction. For other mixed/hybrid schemes for this fourth order elliptic problem, we refer to $[6,7,10-12,29]$.

\section{MiXed VARIATIONAL PROBLEM}

Let $\Omega$ be an open, convex, bounded domain in $\mathbb{R}^{2}$ with Lipschitz-continuous curved boundary $\Gamma$, piecewise of $C^{m}$ class $[1,17,21,32,38] m \geq 3$, in which we consider the boundary value problem $(\mathbf{P})$ : for given $f \in L^{2}(\Omega)$, find $\mathrm{u}$ such that:

$$
(\mathbf{P}): \quad \Lambda u=f \text { in } \Omega,\left.\quad u\right|_{\Gamma}=\left.\left(\frac{\partial u}{\partial n}\right)\right|_{\Gamma}=0
$$

where

$$
(\Lambda u)(x) \equiv \sum_{i=1}^{2} \sum_{j=1}^{2} \sum_{k=1}^{2} \sum_{l=1}^{2} \frac{\partial^{2}}{\partial x_{k} \partial x_{l}}\left(a_{i j k l} \frac{\partial^{2} u}{\partial x_{i} \partial x_{j}}\right)(x) \equiv\left(a_{i j k l} u_{, i j}\right)_{, k l}(x) \quad \forall x \in \bar{\Omega} .
$$

(In (2.2) and also in the sequel, Einstein's summation convention with respect to twice repeated indices $1 \leq i, j, k, l \leq 2$ has been followed), coefficients $a_{i j k l}$ satisfy the following conditions [5]:

$\forall i, j, k, l=1,2$

(A1) $a_{i j k l} \in C^{0}(\bar{\Omega}) ; \quad a_{i j k l} \geq 0 ; a_{i j k l}(x)=a_{k l i j}(x)=a_{j i k l}(x)=a_{j i l k}(x) \forall x \in \bar{\Omega}$;

(A2) $\exists \alpha_{0}>0$ such that $\forall \underline{\xi}=\left(\xi_{11}, \xi_{12}, \xi_{21}, \xi_{22}\right) \in \mathbb{R}^{4}$ with $\xi_{21}=\xi_{12}, \quad a_{i j k l}(x) \xi_{i j} \xi_{k l} \geq \alpha_{0}\|\underline{\xi}\|_{\mathbb{R}^{4}}^{2} \quad \forall x \in \bar{\Omega}$.

Then, under (A1-A2), the corresponding Galerkin variational problem $\left(\mathbf{P}_{\mathbf{G}}\right)$ :

For given $f \in L^{2}(\Omega)$, find $u \in H_{0}^{2}(\Omega)[1,17,21,26,32]$ such that

$$
\left(\mathbf{P}_{\mathbf{G}}\right): a(u, v)=l(v) \quad \forall v \in H_{0}^{2}(\Omega),
$$

where

$$
\begin{array}{rll}
a(u, v)=\langle\Lambda u, v\rangle_{0, \Omega}=\int_{\Omega} a_{i j k l} u_{, i j} v_{, k l} \mathrm{~d} \Omega=a(v, u) & \forall u, v \in H_{0}^{2}(\Omega) ; \\
l(v)=\langle f, v\rangle_{0, \Omega}=\int_{\Omega} f v \mathrm{~d} \Omega & \forall v \in H_{0}^{2}(\Omega)
\end{array}
$$

has a unique solution $[4,20]$.

Introducing Hilbert spaces $\mathbf{H}$ and $\mathbf{V}$ of admissible tensor-valued functions:

- $\mathbf{H}=\left\{\Phi: \Phi=\left(\phi_{i j}\right)_{i, j=1,2} ; \quad \phi_{i j}=\phi_{j i} \in L^{2}(\Omega) \forall i, j=1,2\right\}$

with

$$
\|\Phi\|_{\mathbf{H}}^{2}=\|\Phi\|_{0, \Omega}^{2}=\left\|\phi_{11}\right\|_{0, \Omega}^{2}+2\left\|\phi_{12}\right\|_{0, \Omega}^{2}+\left\|\phi_{22}\right\|_{0, \Omega}^{2} \quad \forall \Phi \in \mathbf{H}
$$


- $\mathbf{V}=\left\{\Phi: \Phi \in \mathbf{H}, \quad \phi_{i j} \in H^{1}(\Omega) \forall i, j=1,2\right\} \subset \mathbf{H}$

with

$$
\|\Phi\|_{\mathbf{V}}^{2}=\|\Phi\|_{1, \Omega}^{2}=\left\|\phi_{11}\right\|_{1, \Omega}^{2}+2\left\|\phi_{12}\right\|_{1, \Omega}^{2}+\left\|\phi_{22}\right\|_{1, \Omega}^{2} \quad \forall \Phi \in \mathbf{V}, \mathbf{V} \hookrightarrow \mathbf{H}
$$

and

- $W \equiv H_{0}^{1}(\Omega)$ with $\|\chi\|_{W}=\|\chi\|_{1, \Omega} \forall \chi \in W$

we associate to $\left(\mathbf{P}_{\mathbf{G}}\right)$, the continuous Mixed Variational Problem $(\mathbf{Q})$ developed in [5] as follows: For given $f \in L^{2}(\Omega)$, find $(\Psi, u) \in \mathbf{V} \times W$ such that

$$
(\mathbf{Q}): \begin{array}{ll}
A(\Psi, \Phi)+b(\Phi, u)=0 & \forall \Phi \in \mathbf{V} \\
-b(\Psi, v)=\langle f, v\rangle_{0, \Omega} & \forall v \in W
\end{array}
$$

where $A(\cdot, \cdot): \mathbf{V} \times \mathbf{V} \longrightarrow \mathbb{R}, b(\cdot, \cdot): \mathbf{V} \times W \longrightarrow \mathbb{R}$ are continuous bilinear forms defined by:

$$
\begin{array}{ll}
A(\Psi, \Phi)=\int_{\Omega} A_{i j k l} \psi_{i j} \phi_{k l} \mathrm{~d} \Omega=A(\Phi, \Psi) & \forall \Psi, \Phi \in \mathbf{V} \subset \mathbf{H} ; \\
b(\Phi, \chi)=\int_{\Omega} \phi_{i j, j} \chi, i \mathrm{~d} \Omega & \forall \Phi \in \mathbf{V}, \forall \chi \in W ;
\end{array}
$$

coefficients $A_{i j k l}=A_{i j k l}(x)$ are defined in terms of $a_{i j k l}$ satisfying the following properties [5]:

- $A_{i j k l} \in C^{0}(\bar{\Omega}), \quad A_{i j k l}(x)=A_{k l i j}(x)=A_{l k i j}(x)=A_{l k j i}(x) \quad \forall i, j, k, l=1,2, \quad \forall x \in \bar{\Omega}$,

- $\exists \alpha_{0}>0$ such that $\forall \underline{\xi}=\left(\xi_{11}, \xi_{12}, \xi_{21}, \xi_{22}\right) \in \mathbb{R}^{4}$ with $\xi_{21}=\xi_{12}, A_{i j k l}(x) \xi_{i j} \xi_{k l} \geq \alpha_{0}\|\underline{\xi}\|_{\mathbb{R}^{4}}^{2} \forall x \in \bar{\Omega}$.

- $\forall x \in \bar{\Omega}, \quad \forall \underline{\xi}=\left(\xi_{11}, \xi_{12}, \xi_{21}, \xi_{22}\right) \in \mathbb{R}^{4}$ with $\xi_{21}=\xi_{12}, \quad \forall \underline{\zeta}=\left(\zeta_{11}, \zeta_{12}, \zeta_{21}, \zeta_{22}\right) \in \mathbb{R}^{4}$

with $\zeta_{21}=\zeta_{12}, \quad A_{i j k l}(x) a_{i j m n}(x) \xi_{m n} \zeta_{k l}=\xi_{i j} \zeta_{i j}$.

Proposition 2.1. [5]

(i) $\exists \alpha>0$ such that

$$
A(\Phi, \Phi) \geq \alpha\|\Phi\|_{\mathbf{H}}^{2} \quad \forall \Phi \in \mathbf{V} \hookrightarrow \mathbf{H}
$$

(ii) $\exists \beta>0$ such that

$$
\sup _{\Phi \in \mathbf{V}-\{\mathbf{0}\}} \frac{|b(\Phi, \chi)|}{\|\Phi\|_{\mathbf{V}}} \geq \beta\|\chi\|_{1, \Omega} \forall \chi \in W
$$

(iii) (Q) has at most one solution $(\Psi, u) \in \mathbf{V} \times W$.

Remark 2.1. (2.17) is Babuška-Brezzi condition $[2,13,14,30]$. 
Since $A(\cdot, \cdot)$ is not $\mathbf{V}$-elliptic, $(\mathbf{Q})$ is not well-posed a priori in general. But we have

Theorem 2.1. [5] If the solution $u \in H_{0}^{2}(\Omega)$ of Galerkin variational problem $\left(\mathbf{P}_{\mathbf{G}}\right)$ belongs to $H^{3}(\Omega) \cap H_{0}^{2}(\Omega)$ and $\psi_{i j}=a_{i j k l} u_{, k l} \in H^{1}(\Omega), \forall i, j=1,2$, then $(\mathbf{Q})$ has a unique solution $(\Psi, u) \in \mathbf{V} \times W$.

Conversely, if $(\mathbf{Q})$ has a solution $(\Psi, u) \in \mathbf{V} \times W$, (which will be a unique one by virtue of Proposition 2.1), the second component $u$ will be the unique solution of $\left(\mathbf{P}_{\mathbf{G}}\right)$ and

$$
\Psi=\left(\psi_{i j}\right)_{i, j=1,2} \text { with } \psi_{i j}=a_{i j k l} u_{, k l} \text { and } u_{, i j}=A_{i j k l} \psi_{k l} \forall i, j=1,2 \text {. }
$$

\section{Examples.}

\section{Biharmonic problem}

For $a_{i j k l}$ defined by: $a_{i i i i}=1 ; a_{1212}=a_{2121}=a_{2112}=a_{1221}=1 / 2 ; a_{i j k l}=0$ otherwise, which satisfy the assumptions (A1-A2) we get the Dirichlet problem of the biharmonic operator $\Lambda \equiv \Delta \Delta$. The coefficients $A_{i j k l}$ are defined by: $A_{i i i i}=1 ; A_{1212}=A_{2121}=A_{2112}=A_{1221}=1 / 2 ; A_{i j k l}=0$ otherwise.

Then, the corresponding bilinear form $A(\cdot, \cdot)$ in $(\mathbf{Q})$ is as follows:

$$
A(\Psi, \Phi)=\int_{\Omega} \psi_{i j} \phi_{i j} \mathrm{~d} \Omega \quad \forall \Psi=\left(\psi_{i j}\right)_{i, j=1,2}, \Phi=\left(\phi_{i j}\right)_{i, j=1,2} \in \mathbf{V} .
$$

In this particular case, the algorithm (Q) reduces to the Hellan-Hermann-Miyoshi (H-H-M) algorithm $[15,28]$ for the biharmonic equation, i.e. the solution $(\Psi, u) \in \mathbf{V} \times W$ of the problem (Q):

$$
\begin{aligned}
\int_{\Omega} \psi_{i j} \phi_{i j} \mathrm{~d} \Omega+\int_{\Omega} \phi_{i j, j} u_{, i} \mathrm{~d} \Omega & =0 & & \forall \Phi \in \mathbf{V}, \\
\int_{\Omega} \psi_{i j, j} v_{, i} \mathrm{~d} \Omega & =-\langle f, v\rangle_{0, \Omega} & & \forall v \in W,
\end{aligned}
$$

is given by: $u, \quad \Psi=\left(\psi_{i j}\right)_{i, j=1,2}$ with $\psi_{i j}=a_{i j k l} u_{, k l}=u_{, i j} \quad \forall i, j=1,2$, where $u \in H_{0}^{2}(\Omega) \cap H^{3}(\Omega)$ is the solution of the problem $\left(\mathbf{P}_{\mathbf{G}}\right)$ corresponding to the biharmonic equation.

Remark 2.2. If $u$ is the deflection of the bent elastic plate, then $\psi_{i j}=u, i j(i, j=1,2)$ denote the components of the change in curvature tensor, but not the bending and twisting moments in the plate in general.

\section{Plate bending problems}

(i) Anisotropic case $[4,25]$ :

$$
\begin{aligned}
& a_{i i i i}=D_{i i}, a_{1212}=a_{1221}=a_{2121}=a_{2112}=D_{66}, a_{1112}=a_{1211}=a_{2111}=a_{1121}=D_{16}, \\
& a_{1222}=a_{2122}=a_{2212}=a_{2221}=D_{26}, a_{2211}=a_{1122}=D_{12}
\end{aligned}
$$

where $D_{i j}=D_{i j}\left(x_{1}, x_{2}\right) \quad \forall\left(x_{1}, x_{2}\right) \in \bar{\Omega}$ denote rigidities [25] defined by $D_{i j}=B_{i j} t^{3} / 12(i=1,2 ; j=1,2,6)$, the $B_{i j}$ 's being expressions in terms of elastic constants of the generalized Hooke's Law for the anisotropic material of the thin plate, $t=t\left(x_{1}, x_{2}\right)$ being the thickness of the plate at the point $\left(x_{1}, x_{2}\right) \in \bar{\Omega}$, such that

$$
\begin{aligned}
& D_{11}, D_{22}, D_{66}>0, D_{12}=\nu_{1} D_{22}=\nu_{2} D_{11}\left(0 \leq \nu_{i}<1 / 2\right), \\
& 0 \leq D_{i 6}<\left(1-\nu_{j}\right) D_{i i}(i \neq j) 1 \leq i, j \leq 2, D_{16}+D_{26}<D_{66} .
\end{aligned}
$$


Define $A_{i j k l}=A_{i j k l}(x) \forall x=\left(x_{1}, x_{2}\right) \in \bar{\Omega} \forall i, j, k, l=1,2$ with the help of $a_{i j k l}$ as follows:

$$
\begin{aligned}
& A_{i i i i}=4\left(D_{j j} D_{66}-D_{j 6}^{2}\right) /|A(\cdot)|(i \neq j) ; A_{1212}=\left(D_{11} D_{22}-D_{12}^{2}\right) /|A(\cdot)| ; \\
& A_{1112}=2\left(D_{12} D_{26}-D_{16} D_{22}\right) /|A(\cdot)| ; A_{1122}=4\left(D_{16} D_{26}-D_{12} D_{66}\right) /|A(\cdot)| ; \\
& A_{1222}=2\left(D_{12} D_{16}-D_{11} D_{26}\right) /|A(\cdot)|
\end{aligned}
$$

with $|A(\cdot)|$ defined by

$$
|A(x)|=4\left(D_{11} D_{22} D_{66}-D_{11} D_{26}^{2}-D_{66} D_{12}^{2}-D_{22} D_{16}^{2}+D_{12} D_{16} D_{26}\right)(x),
$$

and other $A_{i j k l}$ are determined with the symmetry property in $(2.13)$. The corresponding bilinear form $A(\cdot, \cdot)$ in $(\mathrm{Q})$ is given by:

$$
\begin{aligned}
& A(\Psi, \Phi)= \int_{\Omega} \frac{4}{|A(x)|}\left[\left\{\left(D_{22} D_{66}-D_{26}^{2}\right) \psi_{11}+\left(D_{16} D_{26}-D_{12} D_{66}\right) \psi_{22}+\left(D_{12} D_{26}-D_{16} D_{22}\right) \psi_{12}\right\} \phi_{11}\right. \\
&\left\{\left(D_{16} D_{26}-D_{12} D_{66}\right) \psi_{11}+\left(D_{11} D_{66}-D_{16}^{2}\right) \psi_{22}+\left(D_{16} D_{12}-D_{11} D_{26}\right) \psi_{12}\right\} \phi_{22} \\
&\left.\left\{\left(D_{12} D_{26}-D_{16} D_{22}\right) \psi_{11}+\left(D_{16} D_{12}-D_{11} D_{26}\right) \psi_{22}+\left(D_{11} D_{22}-D_{12}^{2}\right) \psi_{12}\right\} \phi_{12}\right] \mathrm{d} \Omega \\
& \forall \Psi, \Phi \in \mathbf{V} ;
\end{aligned}
$$

$b(\cdot, \cdot)$ being the same bilinear form in (2.12).

The solution $(\Psi, u) \in \mathbf{V} \times W$ of $(\mathbf{Q})$ is characterized by: $u$ is the deflection of the bent plate, $\Psi=\left(\psi_{i j}\right)_{1 \leq i, j \leq 2}$ is the bending moment tensor with bending moments $\psi_{i i}$ in the $x_{i}$-direction $(i=1,2)$ and twisting moment $\psi_{12}=\psi_{21}$, i.e. one obtains directly and simultaneously ' $u$ ' and $\psi_{i j}$ 's.

(ii) The orthotropic case $[4,25,37]$ can be obtained from the anisotropic case (i) by putting in $(2.22-2.26)$,

$$
\begin{aligned}
a_{i i i i} & =D_{i} ; a_{1122}=a_{2211}=D_{12}=\nu_{1} D_{2}=\nu_{2} D_{1} \\
a_{1212} & =a_{2121}=a_{2112}=a_{1221}=D_{t}, a_{i j k l}=0 \quad \text { otherwise }
\end{aligned}
$$

where $D_{i}=E_{i} t^{3} /\left(12\left(1-\nu_{1} \nu_{2}\right)\right)>0,(i=1,2) ; D_{\tau}=G t^{3} / 12>0, H=D_{1} \nu_{2}+2 D_{\tau}, G=E_{1} E_{2} /\left(E_{1}+(1+\right.$ $\left.\left.2 \nu_{1}\right) E_{2}\right)>0, E_{1} \nu_{2}=E_{2} \nu_{1}, E_{i}$ and $\nu_{i}, i=1,2$ being the Young's moduli and Poisson's coefficients respectively, and the thickness function $t \in C^{0}(\bar{\Omega})$ is such that $0<t_{0} \leq t\left(x_{1}, x_{2}\right) \leq t_{1}, \forall\left(x_{1}, x_{2}\right) \in \bar{\Omega}$. Then

$$
A(\Psi, \Phi)=\int_{\Omega}\left[\frac{1}{D_{1}\left(1-\nu_{1} \nu_{2}\right)}\left(\psi_{11}-\nu_{1} \psi_{22}\right) \phi_{11}+\frac{1}{D_{2}\left(1-\nu_{1} \nu_{2}\right)}\left(-\nu_{2} \psi_{11}+\psi_{22}\right) \phi_{22}+\frac{1}{D_{\tau}} \psi_{12} \phi_{12}\right] \mathrm{d} \Omega \forall \Psi, \Phi \in \mathbf{V},
$$

and the solution $(\Psi, u) \in \mathbf{V} \times W$ of $(\mathbf{Q})$ is such that $\mathrm{u}$ is the deflection of the bent plate, $\Psi=\left(\psi_{i j}\right)_{i, j=1,2}$ with $\psi_{i j}=a_{i j k l} u_{, k l} \forall i, j=1,2$ giving the bending and twisting moments in the plate, i.e. $\psi_{11}=D_{1}\left(u_{, 11}+\right.$ $\left.\nu_{2} u_{, 22}\right), \psi_{22}=D_{2}\left(\nu_{1} u_{, 11}+u_{, 22}\right)$ are the bending moments in the $x_{1}$ and $x_{2}$ directions, the twisting moment being $\psi_{12}=\psi_{21}=2 D_{t} u, 12$.

(iii) The isotropic case is obtained from the orthotropic case by putting $E_{1}=E_{2}=E$, $\nu_{1}=\nu_{2}=\nu$ and consequently, $D_{1}=D_{2}=D$ in all formulae in (ii) for the orthotropic plate. In this case also, $\mathrm{u}$ is the deflection of the bent plate; $\psi_{11}=D\left(u_{, 11}+\nu u_{, 22}\right), \psi_{22}=D\left(\nu u_{, 11}+u_{, 22}\right), \psi_{12}=\psi_{21}=D(1-\nu) u_{, 12}$ are the bending moments in the $x_{1}$ and $x_{2}$ directions and twisting moment respectively.

Remark 2.3. For $D=1, \nu=0$, we get $\mathbf{H}-\mathbf{H}-\mathbf{M}$ mixed scheme in (2.20-2.21) [15]. 


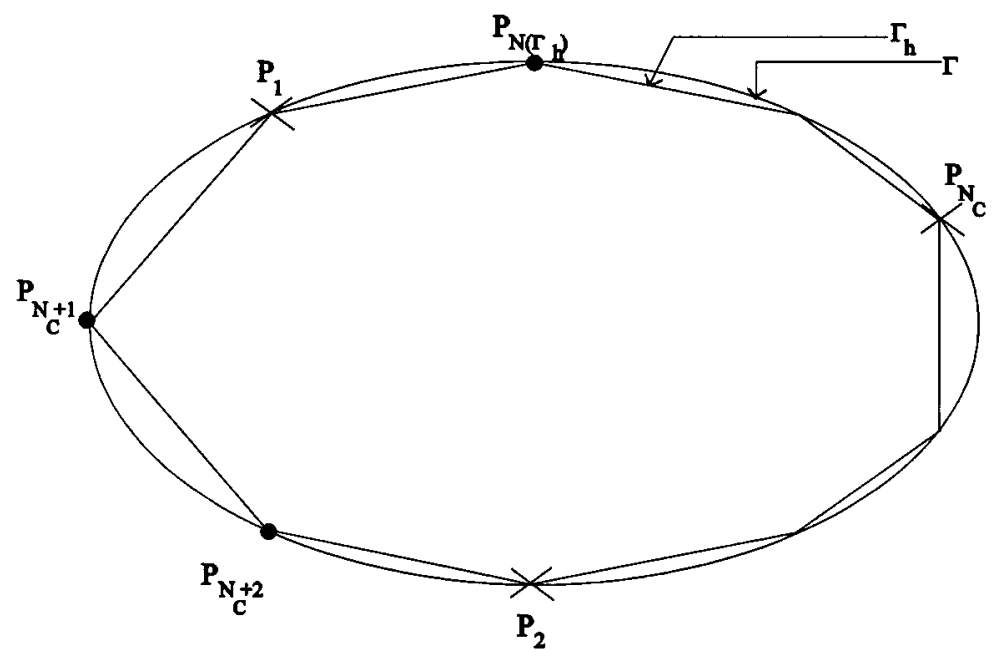

Figure 3.1. $\times$ Points $P_{i} \in \Gamma \cap \Gamma_{h}\left(1 \leq i \leq N_{C}\right)$ at which $C^{m}$ - smoothness does not hold.

- Points $P_{i} \in \Gamma \cap \Gamma_{h}\left(N_{C}+1 \leq i \leq N\left(\Gamma_{h}\right)\right)$ are additional vertices of $\Gamma_{h}$.

\section{Mixed Finite ElEMEnt PROBlem $\left(Q_{h}\right)$ With APPROXimation OF THE CURVED BOUNDARY $\Gamma$ AND NUMERICAL INTEGRATION}

\subsection{Triangulations $\tau_{h}$ and $\tau_{h}^{\text {exact }}$}

Let $\Gamma_{h}$ be a (straight) polygonal boundary approximating $\Gamma$ such that

$$
\Gamma_{h} \subset \bar{\Omega}, \Gamma_{h} \cap \Gamma=\left\{P_{i}\right\}_{i=1}^{N_{c}} \cup\left\{P_{i}\right\}_{i=N_{c}+1}^{N\left(\Gamma_{h}\right)}=V\left(\Gamma_{h}\right)
$$

where $V\left(\Gamma_{h}\right)$ is the set of all vertices (corner points) of $\Gamma_{h}$ with $\operatorname{Card}\left(V\left(\Gamma_{h}\right)\right)=N\left(\Gamma_{h}\right)$, the set of all corner points $\left\{P_{i}\right\}_{i=1}^{N_{c}}$, at which $C^{m}$-smoothness $(m \geq 3)$ does not hold, being its proper subset.

Let $\Omega_{h} \subset \mathbb{R}^{2}$ the domain interior to $\Gamma_{h}$ such that

$$
\bar{\Omega}_{h}=\Omega_{h} \cup \Gamma_{h} \subset \bar{\Omega}
$$

is the closed convex polygonal domain contained in $\bar{\Omega}$ (see Fig. 3.1).

Let $\tau_{h}$ be an exact, admissible, regular, quasi-uniform $[3,17]$ triangulation of $\bar{\Omega}_{h}$ such that

$$
\bar{\Omega}_{h}=\cup_{T \in \tau_{h}} T \subset \bar{\Omega} \text { with } \tau_{h}=\tau_{h}^{b} \cup \tau_{h}^{0},
$$

where

$$
\begin{aligned}
\tau_{h}^{b}= & \left\{T: T \in \tau_{h}, \text { exactly two vertices } a_{1, T} \text { and } a_{2, T} \text { of } T \text { lie on } \Gamma_{h} \cap \Gamma\right. \text { or } \\
& \text { equivalently only one side of } \left.T \text { is a part of } \Gamma_{h}\right\} \\
= & \text { set of all boundary triangles of } \tau_{h} ; \\
\tau_{h}^{0}= & \left\{T: T \in \tau_{h} \text { is an interior triangle } i . e . \text { atmost one of its vertices lie on } \Gamma_{h}\right\} \\
= & \text { set of all interior triangles of } \tau_{h} .
\end{aligned}
$$



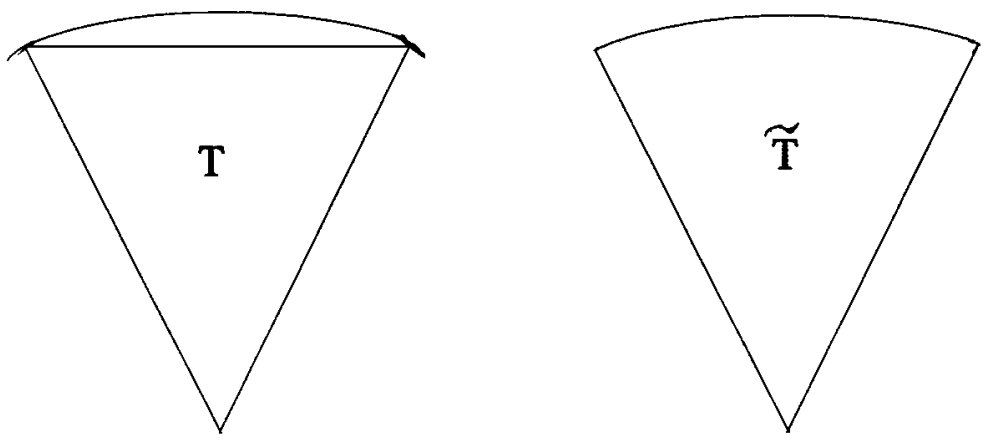

FIGURE 3.2

Let $\widetilde{\tau_{h}^{b}}$ denote the set of all curved boundary triangles $\tilde{T}$ obtained from the boundary triangles $T \in \tau_{h}^{b}$ by replacing the straight boundary side of $T$ by a part of $\Gamma$ joining the two boundary vertices on $\Gamma_{h} \cap \Gamma$, the other two sides being the same ones of the corresponding boundary triangle $T \in \tau_{h}^{b}$. (See Fig. 3.2.)

Then, $\tau_{h}^{\text {exact }}=\widetilde{\tau_{h}^{b}} \cup \tau_{h}^{0}, \tau_{h}^{0} \subset \tau_{h}$ being the set of all interior triangles defined in (3.5), denotes an exact triangulation of $\bar{\Omega}=\Omega \cup \Gamma$. i.e.

$$
\bar{\Omega}=\cup_{\tilde{T} \in \tau_{h}^{\text {exact }}} \tilde{T}, \quad \bar{\Omega}_{h}=\cup_{T \in \tau_{h}} T, \quad \operatorname{Card}\left(\tilde{\tau_{h}^{b}}\right)=\operatorname{Card}\left(\tau_{h}^{b}\right)
$$

\subsection{Reference triangle $\hat{T}$ and affine mapping $F_{T}: \hat{T} \longrightarrow T$}

Let $\hat{T}$ be the reference triangle with vertices $\hat{a}_{1}=(1,0), \hat{a}_{2}=(0,1), \hat{a}_{3}=(0,0)$ and $\forall T \in \tau_{h}, F_{T}: \hat{T} \longrightarrow T$ be an invertible affine mapping from $\hat{T}$ onto $T \in \tau_{h}$ defined by:

$$
\forall \hat{x} \in \hat{T}, F_{T}(\hat{x})=B_{T} \hat{x}+b_{T}=x \in T
$$

such that

$$
\begin{gathered}
F_{T}\left(\hat{a}_{i}\right)=a_{i, T}, 1 \leq i \leq 3,\left\{a_{i, T}\right\}_{i=1}^{3} \text { being the vertices of } T \in \tau_{h}, \\
{\left[J\left(F_{T}\right)\right]=B_{T} \text { is the invertible } 2 \times 2 \text { Jacobian matrix and Jacobian } J\left(F_{T}\right)=\operatorname{det} B_{T}>0,}
\end{gathered}
$$

$\forall \hat{\phi} \in P_{m}(\hat{T}), \exists \phi \in P_{m}(T), P_{m}(K)$ being the linear space of polynomials of degree $\leq m$ defined on $K=\hat{T}$ or $T$, such that $\forall x \in T$ with $x=F_{T}(\hat{x})$,

$$
\phi(x)=\phi \cdot F_{T}(\hat{x})=\hat{\phi}(\hat{x}) \text { with } \hat{\phi}=\phi \cdot F_{T}, \phi=\hat{\phi} \cdot F_{T}^{-1} .
$$

Thus, under the affine mapping $F_{T}$ defined in $(3.7), \tau_{h}$ is affine-equivalent to $\hat{T}$, i.e. $\tau_{h}$ is an affine family of triangles and hence, an exact triangulation of $\bar{\Omega}_{h}=\Omega_{h} \cup \Gamma_{h}$.

\subsection{Numerical integration formulae}

Let

$$
\int_{\hat{T}} \hat{\phi}(\hat{x}) \mathrm{d} \hat{x} \approx \sum_{n=1}^{N_{i}} \hat{w}_{n}^{i} \hat{\phi}\left({\hat{b_{n}}}^{i}\right) \quad(i=1,2)
$$


be two quadrature schemes with positive weights ${\hat{w_{n}}}^{i}>0$ and evaluation points ${\hat{b_{n}}}^{i} \in \hat{T}\left(i=1,2,1 \leq n \leq N_{i}\right)$. The quadrature scheme (3.11) exact for $P_{4}(\hat{T})$ for $i=1$ (resp. $P_{2}(\hat{T})$ for $i=2$ ) will be used in the evaluation of the bilinear forms of the mixed finite element problem in the sequel. Then,

$$
\int_{T} \phi(x) \mathrm{d} x=\int_{\hat{T}} \hat{\phi}(\hat{x}) \operatorname{det}\left(B_{T}\right) \mathrm{d} \hat{x} \approx \sum_{i=1}^{N_{i}} w_{n, T}^{i} \phi\left(b_{n, T}^{i}\right)
$$

with $w_{n, T}^{i}=\operatorname{det}\left(B_{T}\right) \hat{w}_{n}{ }^{i}>0, b_{n, T}^{i}=F_{T}\left({\hat{b_{n}}}^{i}\right) \in T, 1 \leq n \leq N_{i}, \quad i=1,2$, is obtained from (3.11) under invertible affine mapping $F_{T}$ in (3.7-3.10).

To each $\Omega_{h}$, we associate auxiliary infinite dimensional Hilbert spaces $\mathbf{V}\left(\Omega_{h}\right)$ and $H_{0}^{1}\left(\Omega_{h}\right)$ defined by:

- $\mathbf{V}\left(\Omega_{h}\right)=\left\{\Phi: \Phi=\left(\phi_{i j}\right)_{i, j=1,2}, \phi_{i j}=\phi_{j i} \in H^{1}\left(\Omega_{h}\right) \forall i, j=1,2\right\}$

with

$$
\|\Phi\|_{\mathbf{V}\left(\Omega_{h}\right)}^{2}=\|\Phi\|_{1, \Omega_{h}}^{2}=\sum_{i=1}^{2} \sum_{j=1}^{2}\left\|\phi_{i j}\right\|_{1, \Omega_{h}}^{2}
$$

- $H_{0}^{1}\left(\Omega_{h}\right)=\left\{v: v \in H^{1}\left(\Omega_{h}\right),\left.v\right|_{\Gamma_{h}}=0\right\}$ with $\|v\|_{H_{0}^{1}\left(\Omega_{h}\right)}=\|v\|_{1, \Omega_{h}}$,

and the auxiliary continuous bilinear forms

$$
\tilde{A}(\cdot, \cdot): \mathbf{V}\left(\Omega_{h}\right) \times \mathbf{V}\left(\Omega_{h}\right) \longrightarrow \mathbb{R}, \quad \tilde{b}(\cdot, \cdot): \mathbf{V}\left(\Omega_{h}\right) \times H^{1}\left(\Omega_{h}\right) \longrightarrow \mathbb{R}
$$

defined by:

$$
\begin{aligned}
\tilde{A}(\Phi, \Psi)=\int_{\Omega_{h}} A_{i j k l} \phi_{i j} \psi_{k l} \mathrm{~d} \Omega_{h} \text { with }|\tilde{A}(\Psi, \Phi)| \leq \tilde{M}\|\Psi\|_{0, \Omega_{h}}\|\Phi\|_{0, \Omega_{h}} & \forall \Phi, \Psi \in \mathbf{V}\left(\Omega_{h}\right) ; \\
\tilde{b}(\Phi, \chi)=\int_{\Omega_{h}} \phi_{i j, j} \chi, i \mathrm{~d} \Omega_{h} \text { with }|\tilde{b}(\Phi, \chi)| \leq \tilde{m}\|\Phi\|_{1, \Omega_{h}}\|\chi\|_{1, \Omega_{h}} & \forall \Phi \in \mathbf{V}\left(\Omega_{h}\right), \forall \chi \in H^{1}\left(\Omega_{h}\right) .
\end{aligned}
$$

And to each $\tau_{h}$ of $\bar{\Omega}_{h}$, we associate the following finite dimensional subspaces:

- $X_{h}=\left\{\phi_{h}: \phi_{h} \in C^{0}\left(\bar{\Omega}_{h}\right), \phi_{h} \downarrow_{T} \in P_{2}(T) \forall T \in \tau_{h}\right\} \subset H^{1}\left(\Omega_{h}\right)$;

- $\mathbf{V}_{h}=\left\{\Phi_{h}: \Phi_{h}=\left(\phi_{h i j}\right)_{i, j=1,2}, \phi_{h i j}=\phi_{h j i} \in X_{h} \forall i, j=1,2\right\} \subset \mathbf{V}\left(\Omega_{h}\right)$

with

$$
\left\|\Phi_{h}\right\|_{\mathbf{V}_{h}}=\left\|\Phi_{h}\right\|_{\mathbf{V}\left(\Omega_{h}\right)}
$$

- $W_{h}=\left\{\chi_{h}: \chi_{h} \in X_{h}, \chi_{h} \downarrow_{\Gamma_{h}}=0\right\} \subset H_{0}^{1}\left(\Omega_{h}\right)$ with $\left\|\chi_{h}\right\|_{W_{h}}=\left\|\chi_{h}\right\|_{1, \Omega_{h}}$,

in which we have replaced the essential boundary condition $\chi \downarrow_{\Gamma}$ in the definition of $W$ in (2.9) by the boundary condition $\chi_{h} \downarrow_{\Gamma_{h}}$ in (3.19). 


\subsection{Extensions}

Let $\tilde{T} \in \widetilde{\tau_{h}^{b}} \subset \tau_{h}^{\text {exact }}$ be a curved boundary triangle containing the corresponding boundary triangle $T \in$ $\tau_{h}^{b} \subset \tau_{h}$ with $T \subset \tilde{T}$ (see Fig. 3.2). For $\phi_{T}=\phi_{h} \downarrow_{T} \in P_{2}(T)$ with $\phi_{h} \in X_{h}, \tilde{\phi}$ is the natural (polynomial) extension to $\tilde{T}$ of the polynomial $\phi_{T} \in P_{2}(T)$ defined by: $\tilde{\phi} \in P_{2}(\tilde{T})$ with $\tilde{\phi} \downarrow_{T}=\phi_{T} \in P_{2}(T)$.

Then, to $X_{h}$ we associate $\tilde{X}_{h}$ as the linear space of natural (piecewise polynomial) extensions to $\bar{\Omega}$ of functions $\phi_{h} \in X_{h}$ defined in $\bar{\Omega}_{h}$ :

- $\tilde{X}_{h}=\left\{\tilde{\phi}_{h}: \tilde{\phi}_{h} \in C^{0}(\bar{\Omega}), \tilde{\phi}_{h} \downarrow_{\bar{\Omega}_{h}}=\phi_{h} \in X_{h}, \tilde{\phi}_{h} \downarrow_{\tilde{T}} \in P_{2}(\tilde{T}) \forall \tilde{T} \in \widetilde{\tau_{h}^{b}} \subset \tau_{h}^{\text {exact }}\right\} \subset H^{1}(\Omega) ;$

- $\tilde{\mathbf{V}}_{h}=\left\{\tilde{\Phi}_{h}: \tilde{\Phi}_{h}=\left(\widetilde{\phi_{h i j}}\right)_{i, j=1,2}\right.$ with $\widetilde{\phi_{h 12}}=\widetilde{\phi_{h 21}}$ such that $\left.\widetilde{\phi_{h i j}} \in \tilde{X}_{h} \quad \forall i, j=1,2\right\}$;

- $\tilde{W}_{h}=\left\{\tilde{\chi}_{h}: \tilde{\chi}_{h} \downarrow_{\Omega_{h}} \in W_{h}, \tilde{\chi}_{h} \downarrow_{\Omega-\Omega_{h}}=0\right\} \subset H_{0}^{1}(\Omega)$.

With the help of numerical integration formulae in (3.12), we define new continuous, bilinear forms

$$
A_{h}^{N I}(\cdot, \cdot): \mathbf{V}_{h} \times \mathbf{V}_{h} \longrightarrow \mathbb{R}, b_{h}^{N I}(\cdot, \cdot): \mathbf{V}_{h} \times W_{h} \longrightarrow \mathbb{R}
$$

by

$$
A_{h}^{N I}\left(\Phi_{h}, \Psi_{h}\right)=\sum_{T \in \tau_{h}} \sum_{n=1}^{N_{1}} w_{n, T}^{1}\left(A_{i j k l} \phi_{h i j} \psi_{h k l}\right)\left(b_{n, T}^{1}\right)=A_{h}^{N I}\left(\Psi_{h}, \Phi_{h}\right) \quad \forall \Phi_{h}, \Psi_{h} \in \mathbf{V}_{h}
$$

and $\exists M_{0}>0$ such that

$$
\begin{aligned}
\left|A_{h}^{N I}\left(\Psi_{h}, \Phi_{h}\right)\right| & \leq M_{0}\left\|\Psi_{h}\right\|_{0, \Omega_{h}}\left\|\Phi_{h}\right\|_{0, \Omega_{h}} & & \forall \Psi_{h}, \Phi_{h} \in \mathbf{V}_{h} ; \\
b_{h}^{N I}\left(\Phi_{h}, \chi_{h}\right) & =\sum_{T \in \tau_{h}} \sum_{n=1}^{N_{2}} w_{n, T}^{2}\left(\phi_{h i j, j} \chi_{h, i}\right)\left(b_{n, T}^{2}\right) & & \forall \Phi_{h} \in \mathbf{V}_{h}, \forall \chi_{h} \in W_{h},
\end{aligned}
$$

and $\exists m_{0}>0$ such that

$$
\left|b_{h}^{N I}\left(\Phi_{h}, \chi_{h}\right)\right| \leq m_{0}\left\|\Phi_{h}\right\|_{1, \Omega_{h}}\left\|\chi_{h}\right\|_{1, \Omega_{h}} \quad \forall \Phi_{h} \in \mathbf{V}_{h}, \chi_{h} \in W_{h} .
$$

Now, to the problem $(\mathbf{Q})$ in (2.10), we associate the following 'Affine' Mixed Finite Element Problem $\left(\mathbf{Q}_{\mathbf{h}}\right)$ as follows: Find $\left(\Psi_{h}, u_{h}\right) \in \mathbf{V}_{h} \times W_{h}$ such that

$$
\left(\mathbf{Q}_{\mathbf{h}}\right): \begin{array}{ll}
A_{h}^{N I}\left(\Psi_{h}, \Phi_{h}\right)+b_{h}^{N I}\left(\Phi_{h}, u_{h}\right)=0 & \forall \Phi_{h} \in \mathbf{V}_{h}, \\
-b_{h}^{N I}\left(\Psi_{h}, \chi_{h}\right)=\left\langle f, \chi_{h}\right\rangle_{0, \Omega_{h}} & \forall \chi_{h} \in W_{h},
\end{array}
$$

where $A_{h}^{N I}(\cdot, \cdot), b_{h}^{N I}(\cdot, \cdot)$ are defined by $(3.23)$ and $(3.24)$ respectively,

$$
\left\langle f, \chi_{h}\right\rangle_{0, \Omega_{h}}=\int_{\Omega_{h}} f \chi_{h} \mathrm{~d} \Omega_{h} \forall \chi_{h} \in W_{h} .
$$

Remark 3.1. We are considering the important situations in which exact integration of (3.26) is possible. 
Lemma 3.1. Let the quadrature schemes (3.11) with $i=1$ and 2 correspond to the definitions of $A_{h}^{N I}(\cdot, \cdot)$ and $b_{h}^{N I}(\cdot, \cdot)$ in (3.23) and (3.24) respectively.

Then, (a) $\exists \alpha_{0}>0$, independent of $h$, such that

$$
A_{h}^{N I}\left(\Phi_{h}, \Phi_{h}\right) \geq \alpha_{0}\left\|\Phi_{h}\right\|_{0, \Omega_{h}}^{2} \quad \forall \Phi_{h} \in \mathbf{V}_{h}
$$

(b) $\exists \beta_{1}>0$, independent of $h$, such that

$$
\sup _{\Phi_{h} \in \mathbf{V}_{h}-\{0\}} \frac{\left|b_{h}^{N I}\left(\Phi_{h}, \chi_{h}\right)\right|}{\left\|\Phi_{h}\right\|_{\mathbf{V}_{h}}} \geq \beta_{1}\left\|\chi_{h}\right\|_{W_{h}} \forall \chi_{h} \in W_{h} .
$$

Proof. (a) For $i=1$, the quadrature scheme (3.11) used in (3.23) is exact for $P_{4}(\hat{T})$. Then, using (2.14), we have:

$$
\begin{aligned}
\forall T \in \tau_{h}, \quad \sum_{n=1}^{N_{1}} w_{n, T}^{1}\left(A_{i j k l} \phi_{h i j} \phi_{h k l}\right)\left(b_{n, T}^{1}\right) & \geq \alpha_{0} \sum_{n=1}^{N_{1}} w_{n, T}^{1}\left(\phi_{h 11}^{2}\left(b_{n, T}^{1}\right)+2{\phi_{h 12}^{2}}^{2}\left(b_{n, T}^{1}\right)+\phi_{h 22}^{2}\left(b_{n, T}^{1}\right)\right), \\
& =\alpha_{0} \sum_{n=1}^{N_{1}} \hat{w}_{n}{ }^{1}\left(\operatorname{det} B_{T}\right)\left(\hat{\phi}_{11}^{2}\left({\hat{b_{n}}}^{1}\right)+2 \hat{\phi}_{12}^{2}\left({\hat{b_{n}}}^{1}\right)+\hat{\phi}_{22}^{2}\left({\hat{b_{n}}}^{1}\right)\right) \\
& =\alpha_{0} \int_{T}\left(\phi_{h 11}^{2}+2 \phi_{h 12}^{2}+\phi_{h 22}^{2}\right) \mathrm{d} T=\alpha_{0}\left\|\Phi_{h}\right\|_{0, T}^{2} .
\end{aligned}
$$

(b) For $i=2$, the quadrature scheme (3.11) used in (3.24) is exact for $P_{2}(\hat{T})$. Choose $\Phi_{h}^{*}=\left(\chi_{h}, 0,0, \chi_{h}\right)$ with $\chi_{h} \in W_{h}$.

Then

$$
\Phi_{h}^{*} \in \mathbf{V}_{h} \text { with }\left\|\Phi_{h}^{*}\right\|_{1, \Omega_{h}}=\sqrt{2}\left\|\chi_{h}\right\|_{1, \Omega_{h}}
$$

and

$$
\sup _{\Phi_{h} \in \mathbf{V}_{h}-\{0\}} \frac{\left|b_{h}^{N I}\left(\Phi_{h}, \chi_{h}\right)\right|}{\left\|\Phi_{h}\right\|_{1, \Omega_{h}}} \geq \frac{b_{h}^{N I}\left(\Phi_{h}^{*}, \chi_{h}\right)}{\left\|\Phi_{h}^{*}\right\|_{1, \Omega_{h}}}=\frac{b_{h}^{N I}\left(\Phi_{h}^{*}, \chi_{h}\right)}{\sqrt{2}\left\|\chi_{h}\right\|_{1, \Omega_{h}}} \quad \text { [using (3.29)] }
$$

where

$$
\begin{aligned}
b_{h}^{N I}\left(\Phi_{h}^{*}, \chi_{h}\right) & =\sum_{T \in \tau_{h}} \sum_{n=1}^{N_{2}} w_{n, T}^{2}\left[\left(\chi_{h, 1}\right)^{2}+\left(\chi_{h, 2}\right)^{2}\right]\left(b_{n, T}^{2}\right) \geq \gamma \sum_{T \in \tau_{h}}\left|\chi_{h}\right|_{1, T}^{2} \text { with } \gamma>0 \text { [17]. } \\
\Longrightarrow b_{h}^{N I}\left(\Phi_{h}^{*}, \chi_{h}\right) & \geq \gamma \sum_{T \in \tau_{h}}\left|\chi_{h}\right|_{1, T}^{2}=\gamma\left|\chi_{h}\right|_{1, \Omega_{h}}^{2}=\gamma\left|\tilde{\chi_{h}}\right|_{1, \Omega}^{2} .
\end{aligned}
$$

Applying Friedrichs' inequality in (3.31), we have

$$
b_{h}^{N I}\left(\Phi_{h}^{*}, \chi_{h}\right) \geq \gamma C(\Omega)\left\|\tilde{\chi_{h}}\right\|_{1, \Omega}^{2}=\gamma C(\Omega)\left\|\chi_{h}\right\|_{1, \Omega_{h}}^{2} .
$$

From (3.30) and (3.32), we get

$$
\sup _{\Phi_{h} \in \mathbf{V}_{h}-\{0\}} \frac{\left|b_{h}^{N I}\left(\Phi_{h}, \chi_{h}\right)\right|}{\left\|\Phi_{h}\right\|_{\mathbf{V}_{h}}} \geq \beta_{1}\left\|\chi_{h}\right\|_{1, \Omega_{h}} \forall \chi_{h} \in W_{h} \text { with } \beta_{1}=\gamma C(\Omega) / \sqrt{2}>0 .
$$


Remark 3.2. The inequality (3.28) is the discrete Babuška-Brezzi condition $[13,14,30]$.

Theorem 3.1. The 'affine' mixed finite element problem $\left(\mathbf{Q}_{\mathbf{h}}\right)$ defined by (3.25) has a unique solution $\left(\Psi_{h}, u_{h}\right) \in$ $\mathbf{V}_{h} \times W_{h}$.

Proof. Since the linear problem $\left(\mathbf{Q}_{\mathbf{h}}\right)$ is defined on $\mathbf{V}_{h} \times W_{h}$ which is a finite dimensional vector space, the uniqueness of its solution in $\mathbf{V}_{h} \times W_{h}$ implies its existence in $\mathbf{V}_{h} \times W_{h}$. The homogeneous problem corresponding to $\left(\mathbf{Q}_{\mathbf{h}}\right)$ :

$$
\begin{aligned}
A_{h}^{N I}\left(\Psi_{h}, \Phi_{h}\right)+b_{h}^{N I}\left(\Phi_{h}, u_{h}\right)=0 & \forall \Phi_{h} \in \mathbf{V}_{h}, \\
-b_{h}^{N I}\left(\Psi_{h}, \chi_{h}\right)=0 & \forall \chi_{h} \in W_{h},
\end{aligned}
$$

has a unique solution $\Psi_{h}=\mathbf{0}, u_{h}=0$ by virtue of (3.27) and (3.28), from which the result follows.

\section{ERror estimates}

\subsection{Auxiliary interpolation operator $\mathcal{P}_{\boldsymbol{h}}$}

Since functions in $H^{s}(\Omega) \cap H_{0}^{1}(\Omega)$ with $s \geq 2$ are continuous in $\bar{\Omega}$ with $\bar{\Omega}_{h} \subset \bar{\Omega}$ and $\Gamma \cap \Gamma_{h}=V\left(\Gamma_{h}\right)=$ set of boundary vertices of $\tau_{h}=\left\{a_{i, T}\right\}_{i=1, T \in \tau_{h}^{b}}^{2}$ [see (3.1)], we can define an auxiliary interpolation operator $\mathcal{P}_{h}$ as follows: $\forall \chi \in H^{s}(\Omega) \cap H_{0}^{1}(\Omega), s=2,3$,

$$
\mathcal{P}_{h} \chi \in C^{0}\left(\bar{\Omega}_{h}\right), \mathcal{P}_{h} \chi \downarrow_{T} \in P_{2}(T), \mathcal{P}_{h} \chi\left(a_{i, T}\right)=\chi\left(a_{i, T}\right), 1 \leq i \leq 6, \forall T \in \tau_{h},
$$

$\left\{a_{i, T}\right\}_{1}^{3}$ and $\left\{a_{i, T}\right\}_{4}^{6}$ being the vertices and midside nodes of $T \in \tau_{h}$ respectively such that $\partial T_{1}=\left[a_{1, T}, a_{2, T}\right]$ is the boundary side of $T \in \tau_{h}^{b}$. Then, from (4.1) it follows that $\forall$ boundary triangle $T \in \tau_{h}^{b}, \mathcal{P}_{h} \chi\left(a_{i, T}\right)=0(i=1,2)$, but $\mathcal{P}_{h} \chi\left(a_{4, T}\right)=\chi\left(a_{4, T}\right) \neq 0$ in general for $a_{4, T}=\left(a_{1, T}+a_{2, T}\right) / 2$. Hence, $\forall \chi \in H^{s}(\Omega) \cap H_{0}^{1}(\Omega), s=2,3$,

$$
\begin{gathered}
\mathcal{P}_{h} \chi \in\left\{\chi_{h}: \chi_{h} \in H^{1}\left(\Omega_{h}\right) \cap C^{0}\left(\bar{\Omega}_{h}\right), \quad \chi_{h}\left(a_{i, T}\right)=0 \quad \forall T \in \tau_{h}^{b}, i=1,2\right\}, \\
\chi \downarrow_{\Omega_{h}} \in\left\{\chi: \chi \in H^{s}\left(\Omega_{h}\right), \chi\left(a_{i, T}\right)=0 \quad \forall T \in \tau_{h}^{b}, i=1,2\right\},
\end{gathered}
$$

and the classical estimate [17] holds: $\exists C>0$, independent of $h$, such that

$$
\left\|\chi-\mathcal{P}_{h} \chi\right\|_{r, \Omega_{h}} \leq C h^{s-r}|\chi|_{s, \Omega_{h}} \quad(s=2,3 ; r=0,1) .
$$

(In (4.3) and also in the sequel the same $C$ has been used to denote a generic strictly positive constant, independent of $h$, having different values at different steps of the proofs.)

But $\mathcal{P}_{h} \chi \notin W_{h} \subset H_{0}^{1}\left(\Omega_{h}\right)$. Hence, we introduce $W_{h}$ - interpolation operator $\mathcal{P}_{0 h}$ defined by:

$$
\begin{aligned}
& \forall \chi \in H^{s}(\Omega) \cap H_{0}^{1}(\Omega), s=2,3, \quad \mathcal{P}_{0 h} \chi \in C^{0}\left(\bar{\Omega}_{h}\right), \quad \mathcal{P}_{0 h} \chi \downarrow_{T} \in P_{2}(T) \forall T \in \tau_{h}, \\
& \mathcal{P}_{0 h} \chi\left(a_{i, T}\right)=\chi\left(a_{i, T}\right) \quad \forall \text { interior node } a_{i, T} \in \Omega_{h}, \mathcal{P}_{0 h} \chi \downarrow_{\Gamma_{h}}=0 .
\end{aligned}
$$

From (4.4), it follows that $\mathcal{P}_{0 h} \chi \in W_{h} \subset H_{0}^{1}\left(\Omega_{h}\right)$ and we have

Proposition 4.1. Let $\tau_{h}=\tau_{h}^{b} \cup \tau_{h}^{0}$ be the triangulation defined in (3.1-3.5). $\forall \chi \in H^{s}(\Omega) \cap H_{0}^{1}(\Omega), s=2,3$, let $\mathcal{P}_{0 h} \chi \in W_{h}$ be defined by (4.4). Then, the following estimates hold:

$$
\begin{array}{ll}
\text { For } s=2, & \left\|\chi-\mathcal{P}_{0 h} \chi\right\|_{r, \Omega_{h}} \leq C h^{2-r}\|\chi\|_{2, \Omega} \quad(r=0,1) ; \\
\text { For } s=3, & \left\|\chi-\mathcal{P}_{0 h} \chi\right\|_{r, \Omega_{h}} \leq C h^{3-r-1 / 2}\|\chi\|_{3, \Omega} \quad(r=0,1) .
\end{array}
$$


Proof. $\forall \chi \in H^{s}(\Omega) \cap H_{0}^{1}(\Omega), s=2,3$,

$$
\left\|\chi-\mathcal{P}_{0 h} \chi\right\|_{0, \Omega_{h}} \leq\left\|\chi-\mathcal{P}_{h} \chi\right\|_{0, \Omega_{h}}+\left\|\mathcal{P}_{h} \chi-\mathcal{P}_{0 h} \chi\right\|_{0, \Omega_{h}}
$$

and

$$
\left|\chi-\mathcal{P}_{0 h} \chi\right|_{1, \Omega_{h}} \leq\left|\chi-\mathcal{P}_{h} \chi\right|_{1, \Omega_{h}}+\left|\mathcal{P}_{h} \chi-\mathcal{P}_{0 h} \chi\right|_{1, \Omega_{h}},
$$

where $\mathcal{P}_{h} \chi$ is defined by (4.1). Then, from (4.3),

$$
\left\|\chi-\mathcal{P}_{h} \chi\right\|_{0, \Omega_{h}} \leq C h^{s}|\chi|_{s, \Omega_{h}} ; \quad\left\|\chi-\mathcal{P}_{h} \chi\right\|_{1, \Omega_{h}} \leq C h^{s-1}|\chi|_{s, \Omega_{h}} .
$$

From $(4.1,4.3,4.4)$, we have: $\forall$ interior triangle $T \in \tau_{h}^{0},\left(\mathcal{P}_{h} \chi-\mathcal{P}_{0 h} \chi\right) \downarrow_{T}=0$, and $\forall$ boundary triangle $T \in \tau_{h}^{b},\left(\mathcal{P}_{h} \chi-\mathcal{P}_{0 h} \chi\right) \downarrow_{T}=\chi\left(a_{4, T}\right) \phi_{4, T}$ with $\phi_{4, T} \in P_{2}(T), \phi_{4, T}\left(a_{4, T}\right)=1, \phi_{4, T}\left(a_{i, T}\right)=0, \quad 1 \leq i \neq 4 \leq$ $6, a_{4, T}=\left(a_{1, T}+a_{2, T}\right) / 2$ being the midpoint of the boundary side $\partial T_{1}$ of $T \in \tau_{h}^{b}$.

Hence,

$$
\left\|\mathcal{P}_{h} \chi-\mathcal{P}_{0 h} \chi\right\|_{0, \Omega_{h}}^{2}=\sum_{T \in \tau_{h}^{b}}\left\|\mathcal{P}_{h} \chi-\mathcal{P}_{0 h} \chi\right\|_{0, T}^{2}=\sum_{T \in \tau_{h}^{b}}\left|\chi\left(a_{4, T}\right)\right|^{2}\left\|\phi_{4, T}\right\|_{0, T}^{2}
$$

and

$$
\left|\mathcal{P}_{h} \chi-\mathcal{P}_{0 h} \chi\right|_{1, \Omega_{h}}^{2}=\sum_{T \in \tau_{h}^{b}}\left|\chi\left(a_{4, T}\right)\right|^{2}\left|\phi_{4, T}\right|_{1, T}^{2}
$$

But

$$
\left\|\phi_{4, T}\right\|_{0, T}^{2} \leq C h_{T}^{2}\left\|\hat{\phi}_{4}\right\|_{0, \hat{T}}^{2} \leq C h_{T}^{2} ; \quad\left|\phi_{4, T}\right|_{1, T}^{2} \leq C\left|\hat{\phi}_{4}\right|_{1, \hat{T}}^{2} \leq C \quad[16,38] .
$$

Now, we will find estimate for $\left|\chi\left(a_{4, T}\right)\right|$ in (4.10) and (4.11), for which we are to consider the cases $s=2$ and $s=3$ separately.

Case $s=2$. From imbedding results $[1] H^{2}(\Omega) \hookrightarrow C^{0, \lambda}(\bar{\Omega})$ with $\lambda \in\left[0,1\left[, C^{0, \lambda}(\bar{\Omega})\right.\right.$ being the linear space of $\lambda$-Holder continuous functions in $\Omega$. Hence, $\forall \chi \in H^{2}(\Omega) \cap H_{0}^{1}(\Omega) \subset C^{0, \lambda}(\bar{\Omega})$, with $\lambda \in\left[0,1\left[, \mid \chi\left(a_{4, T}\right)-\right.\right.$ $\chi\left(\tilde{a}_{4, T}\right) \mid \leq C\left\|a_{4, T}-\tilde{a}_{4, T}\right\|_{\mathbb{R}^{2}}^{\lambda}\|\chi\|_{2, \Omega}$, where $\tilde{a}_{4, T} \in \Gamma$ is the point of intersection of the perpendicular bisector of the boundary side $\partial T_{1}=\left[a_{1, T}, a_{2, T}\right]$ of $T \in \tau_{h}^{b}$ with the boundary $\partial \tilde{T} \cap \Gamma$ such that $\left\|a_{4, T}-\tilde{a}_{4, T}\right\|_{\mathbb{R}^{2}} \leq C h_{T}^{2}$ and $\chi\left(\tilde{a}_{4, T}\right)=0$.

Hence

$$
\left|\chi\left(a_{4, T}\right)\right| \leq C h_{T}^{2 \lambda}\|\chi\|_{2, \Omega} \forall \lambda \subset[0,1[\text {. }
$$

From (4.10) and (4.12), for $h=\max _{T \in \tau_{h}}\left\{h_{T}\right\}$, we have

$$
\begin{aligned}
\left\|\mathcal{P}_{h} \chi-\mathcal{P}_{0 h} \chi\right\|_{0, \Omega_{h}}^{2} & \leq C\left[\sum_{T \in \tau_{h}^{b}} h_{T}^{4 \lambda}\|\chi\|_{2, \Omega}^{2} h_{T}^{2}\right] \leq C h^{4 \lambda+1}\left(\sum_{T \in \tau_{h}^{b}} h_{T}\right)\|\chi\|_{2, \Omega}^{2} \\
& \leq C h^{4 \lambda+1} \operatorname{meas}\left(\Gamma_{h}\right)\|\chi\|_{2, \Omega}^{2} \leq C h^{4 \lambda+1} \operatorname{meas}(\Gamma)\|\chi\|_{2, \Omega}^{2} \leq C h^{4 \lambda+1}\|\chi\|_{2, \Omega}^{2},
\end{aligned}
$$

where $\sum_{T \in \tau_{h}^{b}} h_{T} \leq C$ meas $\left(\Gamma_{h}\right)$ for some $C>0$, independent of ' $h^{\prime}$ ', since $\tau_{h}$ is a regular triangulation.

$$
\Longrightarrow\left\|\mathcal{P}_{h} \chi-\mathcal{P}_{0 h} \chi\right\|_{0, \Omega_{h}} \leq C h^{2 \lambda+1 / 2}\|\chi\|_{2, \Omega} \text { with } \lambda \in[0,1[\text {. }
$$


Similarly, from (4.11-4.13), we get

$$
\begin{aligned}
\left|\mathcal{P}_{h} \chi-\mathcal{P}_{0 h} \chi\right|_{1, \Omega_{h}}^{2} & \leq C \sum_{T \in \tau_{h}^{b}} h_{T}^{4 \lambda}\|\chi\|_{2, \Omega}^{2} \\
& \leq C h^{4 \lambda-1}\left(\sum_{T \in \tau_{h}^{b}} h_{T}\right)\|\chi\|_{2, \Omega}^{2} \\
& \left.\leq C h^{4 \lambda-1} \operatorname{meas}(\Gamma)\|\chi\|_{2, \Omega}^{2} \text { with } \lambda \in\right] 1 / 4,1[ \\
\Longrightarrow\left|\mathcal{P}_{h} \chi-\mathcal{P}_{0 h} \chi\right|_{1, \Omega_{h}} & \left.\leq C h^{2 \lambda-1 / 2}\|\chi\|_{2, \Omega} \text { with } \lambda \in\right] 1 / 4,1[.
\end{aligned}
$$

Hence, from $(4.7-4.9,4.14,4.15)$, we get: for $\lambda \in[3 / 4,1[$,

$$
\begin{aligned}
\left\|\chi-\mathcal{P}_{0 h} \chi\right\|_{0, \Omega_{h}} & \leq C\left[h^{2}|\chi|_{2, \Omega_{h}}+h^{2 \lambda+1 / 2}\|\chi\|_{2, \Omega}\right] \leq C h^{2}\|\chi\|_{2, \Omega} \\
\left|\chi-\mathcal{P}_{0 h} \chi\right|_{1, \Omega_{h}} & \leq C\left[h|\chi|_{2, \Omega_{h}}+h^{2 \lambda-1 / 2}\|\chi\|_{2, \Omega}\right] \leq C h\|\chi\|_{2, \Omega}
\end{aligned}
$$

and

$$
\left\|\chi-\mathcal{P}_{0 h} \chi\right\|_{1, \Omega_{h}}^{2}=\left|\chi-\mathcal{P}_{0 h} \chi\right|_{1, \Omega_{h}}^{2}+\left\|\chi-\mathcal{P}_{0 h} \chi\right\|_{0, \Omega_{h}}^{2} \leq C h^{2}\|\chi\|_{2, \Omega}^{2}
$$

which implies the result.

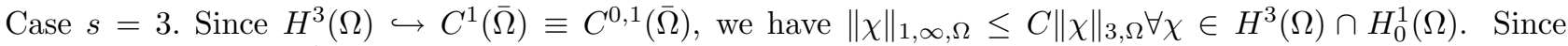
$\left\|a_{4, T}-\tilde{a}_{4, T}\right\|_{\mathbb{R}^{2}} \leq C h_{T}^{2}$, using the mean-value theorem along the line segment $\left[\tilde{a}_{4, T}, a_{4, T}\right]$ we have:

$$
\forall \chi \in H^{3}(\Omega) \cap H_{0}^{1}(\Omega),\left|\chi\left(a_{4, T}\right)\right| \leq C h_{T}^{2} \sup _{\vec{\xi} \in] \tilde{a}_{4, T}, a_{4, T}[}\left|\frac{\partial \chi}{\partial s}(\vec{\xi})\right| \leq C h_{T}^{2}|\chi|_{1, \infty, \Omega} \leq C h_{T}^{2}\|\chi\|_{3, \Omega} .
$$

Hence, from $(4.10-4.12,4.16)$,

$$
\begin{aligned}
\left\|\mathcal{P}_{h} \chi-\mathcal{P}_{0 h} \chi\right\|_{0, \Omega_{h}}^{2} & \leq C\left[\sum_{T \in \tau_{h}^{b}} h_{T}^{4}\|\chi\|_{3, \Omega}^{2} h_{T}^{2}\right] \leq C h^{5}\left(\sum_{T \in \tau_{h}^{b}} h_{T}\right)\|\chi\|_{3, \Omega}^{2} \\
\Longrightarrow\left\|\mathcal{P}_{h} \chi-\mathcal{P}_{0 h} \chi\right\|_{0, \Omega_{h}} & \leq C h^{5 / 2}(\operatorname{meas}(\Gamma))^{1 / 2}\|\chi\|_{3, \Omega} \leq C h^{5 / 2}\|\chi\|_{3, \Omega}
\end{aligned}
$$

and

$$
\begin{aligned}
\left|\mathcal{P}_{h} \chi-\mathcal{P}_{0 h} \chi\right|_{1, \Omega_{h}}^{2} & \leq C\left[\sum_{T \in \tau_{h}^{b}} h_{T}^{4}\|\chi\|_{3, \Omega}^{2}\right] \leq C h^{3}\left(\sum_{T \in \tau_{h}^{b}} h_{T}\right)\|\chi\|_{3, \Omega}^{2} \\
\Longrightarrow\left|\mathcal{P}_{h} \chi-\mathcal{P}_{0 h} \chi\right|_{1, \Omega_{h}} & \leq C h^{3 / 2}(\operatorname{meas}(\Gamma))^{1 / 2}\|\chi\|_{3, \Omega} \leq C h^{3 / 2}\|\chi\|_{3, \Omega} .
\end{aligned}
$$


Thus, from (4.7-4.9) and (4.17-4.18), we get (4.5-4.6):

$$
\begin{aligned}
\left\|\chi-\mathcal{P}_{0 h} \chi\right\|_{0, \Omega_{h}} & \leq C\left[h^{3}|\chi|_{3, \Omega_{h}}+h^{5 / 2}\|\chi\|_{3, \Omega}\right] \leq C h^{5 / 2}\|\chi\|_{3, \Omega} \\
\left|\chi-\mathcal{P}_{0 h} \chi\right|_{1, \Omega_{h}} & \leq C\left[h^{2}|\chi|_{3, \Omega_{h}}+h^{3 / 2}\|\chi\|_{3, \Omega}\right] \leq C h^{3 / 2}\|\chi\|_{3, \Omega}
\end{aligned}
$$

and

$$
\left\|\chi-\mathcal{P}_{0 h} \chi\right\|_{1, \Omega_{h}}^{2}=\left|\chi-\mathcal{P}_{0 h} \chi\right|_{1, \Omega_{h}}^{2}+\left\|\chi-\mathcal{P}_{0 h} \chi\right\|_{0, \Omega_{h}}^{2} \leq C h^{3}\|\chi\|_{3, \Omega}^{2}
$$

and we get (4.6).

Remark 4.1. There is a loss of exponent of $h$ by $1 / 2$ in (4.6) due to a 'crude' polygonal approximation of the curved boundary $\Gamma$. Moreover, from the proof of the Case $s=3$, we find that it can not be improved upon even by assuming additional regularity of $\chi$ i.e. $\left\|\chi-\mathcal{P}_{0 h} \chi\right\|_{r, \Omega_{h}} \leq C h^{3-r-1 / 2}\|\chi\|_{3, \Omega} \forall \chi \in H^{s}(\Omega) \cap H_{0}^{1}(\Omega)$ with $s>3$. Hence it suggests to improve the boundary approximation, for example, by isoparametric mapping [9].

We will need the inverse inequalities [14,17,18]: $\forall \phi_{h} \in X_{h}$ (resp. $\Phi_{h} \in \mathbf{V}_{h}$ ), $\exists \gamma^{*}>0$ (resp. $\exists \gamma>0$ ) independent of $h$, such that

$$
\left|\phi_{h}\right|_{1, \Omega_{h}} \leq \frac{\gamma^{*}}{h}\left|\phi_{h}\right|_{0, \Omega_{h}} \quad\left(\text { resp. }\left|\Phi_{h}\right|_{1, \Omega_{h}} \leq \frac{\gamma}{h}\left|\Phi_{h}\right|_{0, \Omega_{h}}\right)
$$

and the following important well known estimates:

Proposition 4.2. [38] For domains $\Omega$ and $\Omega_{h}$ defined earlier such that $\omega_{h}=\Omega-\Omega_{h}$ with $\left.h \in\right] 0, h_{0}\left[, 0<h_{0}<\right.$ $1, \forall \chi \in H^{1}(\Omega)$,

$$
\|\chi\|_{0, \omega_{h}} \leq C h\|\chi\|_{1, \Omega} \quad \text { for some } C>0 .
$$

Lemma 4.1 (p. $199[36])$. Let $T \in \tau_{h}^{b}$ and $\tilde{T} \in \widetilde{\tau_{h}^{b}}$ be any pair of boundary triangles such that $T \subset \tilde{T}, \tilde{T} \in \tau_{h}^{\text {exact }}$ being the curved boundary triangle constructed from the boundary triangle $T \in \tau_{h}$ [see (3.1-3.6)]. Suppose that $\rho=$ meas $(\tilde{T}-T) /$ meas $T$. Let $\tilde{p}$ be a polynomial on $\tilde{T}$, which is a natural (polynomial) extension to $\tilde{T}$ of the polynomial ' $p$ ' defined on $T$. Then, $\exists C>0$, depending only on the degree of $p$, such that

$$
\|\tilde{p}\|_{1, \tilde{T}-T}^{2} \leq C \rho(T)\|p\|_{1, T}^{2} \quad \forall T \in \tau_{h}^{b} \subset \tau_{h} .
$$

Corollary 4.1. Let $\tilde{\phi}_{h} \in \tilde{X}_{h}$ be the natural extension to $\bar{\Omega}$ of the function $\phi_{h} \in X_{h}$ defined in (3.20). Then, $\exists C>0$, independent of $h$, such that

$$
\left\|\tilde{\phi}_{h}\right\|_{1, \omega_{h}}^{2}=\sum_{T \subset \tilde{T} \in \tilde{\tau}_{h}^{b}}\left\|\tilde{\phi}_{h}\right\|_{1, \tilde{T}-T}^{2} \leq C h\left\|\phi_{h}\right\|_{1, \Omega_{h}}^{2} \forall \phi_{h} \in X_{h} \text { with } \tilde{\phi}_{h} \in \tilde{X}_{h}
$$

and

$$
\omega_{h}=\Omega-\Omega_{h}, \operatorname{meas}\left(\omega_{h}\right)=O\left(h^{2}\right)[38] .
$$

Proof. The result (4.23) is obtained from (4.22) by summing over all boundary triangles $T \in \tau_{h}^{b}$ with $T \subset$ $\tilde{T} \in \widetilde{\tau_{h}^{b}}$ and increasing the right-hand side to include all interior triangles $T \in \tau_{h}^{0}$ and considering the fact that $\rho=O(h) \forall \tau_{h}[36]$. 


\section{Proposition 4.3.}

- Let $A_{i j k l} \in W^{1, \infty}(\Omega) \forall i, j, k, l=1,2$.

- Let the quadrature scheme (3.11) with $i=1$, which is exact for $P_{4}(\hat{T})$, correspond to the definition (3.23) of $A_{h}^{N I}(\cdot, \cdot)$. Then, $\exists C>0$, independent of $h$, such that $\forall \underline{\sigma}_{h}, \Phi_{h} \in \mathbf{V}_{h}$,

$$
\left|\tilde{A}\left(\underline{\sigma}_{h}, \Phi_{h}\right)-A_{h}^{N I}\left(\underline{\sigma}_{h}, \Phi_{h}\right)\right| \leq C h\|A\|_{1, \infty, \Omega}\left\|\underline{\sigma}_{h}\right\|_{0, \Omega_{h}}\left\|\Phi_{h}\right\|_{0, \Omega_{h}},
$$

where $\tilde{A}(\cdot, \cdot)$ and $A_{h}^{N I}(\cdot, \cdot)$ are defined by (3.15) and (3.23) respectively,

$$
\|A\|_{1, \infty, \Omega}=\sup _{\tilde{T} \in \tau_{h}^{\text {exact }}} \sum_{i, j, k, l=1}^{2}\left\|A_{i j k l}\right\|_{1, \infty, \tilde{T}}
$$

Proof. For fixed $i, j, k, l=1,2$ (i.e. no summation is to be understood with respect to twice repeated indices $i, j, k, l), \forall T \in \tau_{h}$, set

$$
\begin{aligned}
E_{T}\left(A_{i j k l} \sigma_{h i j} \phi_{h k l}\right) & =\int_{T} A_{i j k l} \sigma_{h i j} \phi_{h k l} \mathrm{~d} T-\sum_{n=1}^{N_{1}} w_{n, T}^{1}\left(A_{i j k l} \sigma_{h i j} \phi_{h k l}\right)\left(b_{n, T}^{1}\right), \\
\hat{E}\left(\widehat{A_{i j k l}} \hat{\sigma_{i j}} \hat{\phi_{k l}}\right) & =\int_{\hat{T}} \widehat{A_{i j k l}}(\hat{x}) \hat{\sigma_{i j}}(\hat{x}) \hat{\phi_{k l}}(\hat{x}) \mathrm{d} \hat{T}-\sum_{n=1}^{N_{1}} \hat{w_{n}^{1}}\left(\widehat{A_{i j k l}} \hat{\sigma_{i j}} \hat{\phi_{k l}}\right)\left(\hat{b_{n}^{1}}\right)
\end{aligned}
$$

with

$$
E_{T}\left(A_{i j k l} \sigma_{h i j} \phi_{h k l}\right)=\left(\operatorname{det} B_{T}\right) \hat{E}\left(\widehat{A_{i j k l}} \hat{\sigma_{i j}} \hat{\phi_{k l}}\right), b_{n, T}^{1}=F_{T}\left(\hat{b_{n}^{1}}\right)
$$

Then

$$
\begin{aligned}
\left|\tilde{A}\left(\underline{\sigma}_{h}, \Phi_{h}\right)-A_{h}^{N I}\left(\underline{\sigma}_{h}, \Phi_{h}\right)\right| & =\left|\int_{\Omega_{h}} A_{i j k l} \sigma_{h i j} \phi_{h k l} \mathrm{~d} \Omega_{h}-\sum_{T \in \tau_{h}} \sum_{n=1}^{N_{1}} w_{n, T}^{1}\left(A_{i j k l} \sigma_{h i j} \phi_{h k l}\right)\left(b_{n, T}^{1}\right)\right| \\
& \leq \sum_{T \in \tau_{h}} \sum_{i, j, k, l=1}^{2}\left|E_{T}\left(A_{i j k l} \sigma_{h i j} \phi_{h k l}\right)\right| .
\end{aligned}
$$

$\forall$ fixed $i, j, k, l=1,2, \hat{\sigma_{i j}}, \hat{\phi_{k l}} \in P_{2}(\hat{T}), \widehat{A_{i j k l}} \in W^{1, \infty}(\hat{T})$ and hence

$$
\begin{aligned}
\left|\hat{E}\left(\widehat{A_{i j k l}} \hat{\sigma_{i j}} \hat{\phi_{k l}}\right)\right| & \leq C\left\|\widehat{A_{i j k l}} \hat{\sigma_{i j}} \hat{\phi_{k l}}\right\|_{0, \infty, \hat{T}} \leq C\left\|\widehat{A_{i j k l}}\right\|_{0, \infty, \hat{T}}\left\|\hat{\sigma_{i j}} \hat{\phi_{k l}}\right\|_{0, \infty, \hat{T}} \\
& \leq C\left\|\widehat{A_{i j k l}}\right\|_{1, \infty, \hat{T}}\left\|\hat{\sigma_{i j} \hat{\phi_{k l}}}\right\|_{0, \infty, \hat{T}} .
\end{aligned}
$$

$\forall$ fixed $i, j, k, l=1,2$, and for fixed $\hat{\sigma_{i j}}, \hat{\phi_{k l}} \in P_{2}(\hat{T})$, define

$$
\hat{\mathcal{E}}(\cdot): W^{1, \infty}(\hat{T}) \longrightarrow \mathbb{R} \text { by } \hat{\mathcal{E}}\left(\widehat{A_{i j k l}}\right)=\hat{E}\left(\widehat{A_{i j k l}} \hat{\sigma_{i j}} \hat{\phi_{k l}}\right) \text {. }
$$

From $(4.32),(4.33) \hat{\mathcal{E}}(\cdot)$ is a linear bounded functional on $W^{1, \infty}(\hat{T})$ with $\|\hat{\mathcal{E}}(\cdot)\| \leq C\left\|\hat{\sigma_{i j}} \hat{\phi_{k l}}\right\|_{0, \infty, \hat{T}}$ and $\hat{\mathcal{E}}\left(\hat{p_{0}}\right)=$ $\hat{E}\left(\hat{p_{0}} \hat{\sigma_{i j}} \hat{\phi_{k l}}\right)=0 \forall \hat{p_{0}} \in P_{0}(\hat{T})$, since the quadrature formula in $(4.29)$ is exact for $P_{4}(\hat{T})$. 
Hence, by Bramble-Hilbert lemma, we have: $\forall$ fixed $i, j, k, l=1,2$ (with no summation),

$$
\left|\hat{\mathcal{E}}\left(\widehat{A_{i j k l}}\right)\right|=\left|\hat{E}\left(\widehat{A_{i j k l}} \hat{\sigma_{i j}} \hat{\phi_{k l}}\right)\right| \leq C\left\|\hat{\sigma_{i j}} \hat{\phi_{k l}}\right\|_{0, \infty, \hat{T}}\left|\widehat{A_{i j k l}}\right|_{1, \infty, \hat{T}}
$$

But $\forall$ fixed $T \in \tau_{h}$,

$$
\left|\widehat{A_{i j k l}}\right|_{1, \infty, \hat{T}} \leq C h_{T}\left\|A_{i j k l}\right\|_{1, \infty, T} \forall i, j, k, l=1,2 \quad[16]
$$

and

$$
\begin{aligned}
\left\|\hat{\sigma_{i j}} \hat{\phi_{k l}}\right\|_{0, \infty, \hat{T}} & \leq\left\|\hat{\sigma_{i j}}\right\|_{0, \infty, \hat{T}}\left\|\hat{\phi_{k l}}\right\|_{0, \infty, \hat{T}} \leq C\left\|\hat{\sigma_{i j}}\right\|_{0, \hat{T}}\left\|\hat{\phi_{k l}}\right\|_{0, \hat{T}} \quad \text { (norm equivalence in a f.d.v.s.) } \\
& \leq C\left(\operatorname{det} B_{T}\right)^{-1}\left\|\sigma_{h i j}\right\|_{0, T}\left\|\phi_{h k l}\right\|_{0, T}[16] .
\end{aligned}
$$

Hence, $\forall$ fixed $i, j, k, l=1,2$,

$$
\begin{aligned}
\left|\hat{E}\left(\left(\widehat{A_{i j k l}}\right)\left(\hat{\sigma_{i j}} \hat{\phi_{k l}}\right)\right)\right| & \leq C h_{T}\left(\operatorname{det} B_{T}\right)^{-1}\left\|\sigma_{h i j}\right\|_{0, T}\left\|\phi_{h k l}\right\|_{0, T}\left\|A_{i j k l}\right\|_{1, \infty, T} \quad \forall T \in \tau_{h} . \\
\Longrightarrow\left|E_{T}\left(A_{i j k l} \sigma_{h i j} \phi_{h k l}\right)\right| & \leq C h_{T}\left\|\sigma_{h i j}\right\|_{0, T}\left\|\phi_{h k l}\right\|_{0, T}\left\|A_{i j k l}\right\|_{1, \infty, T} \quad \forall T \in \tau_{h} \quad[\text { using }(4.30)] \\
\Longrightarrow \sum_{T \in \tau_{h}} \sum_{i, j, k, l=1}^{2}\left|E_{T}\left(A_{i j k l} \sigma_{h i j} \phi_{h k l}\right)\right| & \leq \sum_{T \in \tau_{h}} \sum_{i, j, k, l=1}^{2} C h_{T}\left\|\sigma_{h i j}\right\|_{0, T}\left\|\phi_{h k l}\right\|_{0, T}\left\|A_{i j k l}\right\|_{1, \infty, T} \\
& \leq C h\left(\sum_{T \in \tau_{h}} \sum_{i, j, k, l=1}^{2}\left\|A_{i j k l}\right\|_{1, \infty, T}\right)\left(\sum_{T \in \tau_{h}} \sum_{i, j, k, l=1}^{2}\left\|\sigma_{h i j}\right\|_{0, T}\left\|\phi_{h k l}\right\|_{0, T}\right) \\
& \leq C h\|A\|_{1, \infty, \Omega}\left\|\underline{\sigma}_{h}\right\|_{0, \Omega_{h}}\left\|\Phi_{h}\right\|_{0, \Omega_{h}}
\end{aligned}
$$

where

$$
\|A\|_{1, \infty, \Omega} \geq\|A\|_{1, \infty, \Omega_{h}}=\sup _{T \in \tau_{h}} \sum_{i, j, k, l=1}^{2}\left\|A_{i j k l}\right\|_{1, \infty, T}
$$

and the result (4.26) follows from $(4.31,4.39,4.40)$.

Proposition 4.4. Suppose that the conditions of Theorem 2.1 hold. Then,

$$
\left|\tilde{A}\left(\Psi, \Phi_{h}\right)+\tilde{b}\left(\Phi_{h}, u\right)\right| \leq C h^{\frac{3}{2}}(1+\sqrt{h})\|u\|_{3, \Omega}\left\|\Phi_{h}\right\|_{1, \Omega_{h}},
$$

where $\tilde{A}(\cdot, \cdot)$ and $\tilde{b}(\cdot, \cdot)$ are defined by (3.15) and (3.16) respectively.

Proof. From the conditions of Theorem 2.1, $u \in H^{3}(\Omega) \cap H_{0}^{2}(\Omega)$ is the solution of $\left(\mathbf{P}_{\mathbf{G}}\right)$ with $\Psi=\left(\psi_{i j}\right)_{i, j=1,2}$, $\psi_{i j}=a_{i j k l} u, k l \in H^{1}(\Omega) \quad \forall i, j=1,2$. 
Then, $\forall \Phi_{h} \in \mathbf{V}_{h}$

$$
\begin{aligned}
\left|\tilde{A}\left(\Psi, \Phi_{h}\right)+\tilde{b}\left(\Phi_{h}, u\right)\right| & =\left|\int_{\Omega_{h}} A_{i j k l} \psi_{i j} \phi_{h k l} \mathrm{~d} x+\int_{\Omega_{h}} \phi_{h i j, j} u_{, i} \mathrm{~d} x\right| \\
& =\left|\int_{\Omega_{h}} A_{i j k l} \psi_{i j} \phi_{h k l} \mathrm{~d} x+\int_{\Omega_{h}} \phi_{h i j, j} u_{, i} \mathrm{~d} x-\int_{\Omega} A_{i j k l} \psi_{i j} \widetilde{\phi_{h k l} \mathrm{~d}} \mathrm{~d}-\int_{\Omega} \widetilde{\phi_{h i j, j}} u_{, i} \mathrm{~d} x\right|
\end{aligned}
$$

[by virtue of (2.10)]

$$
\leq\left|\int_{\omega_{h}} A_{i j k l} \psi_{i j} \widetilde{\phi_{h k l}} d x\right|+\left|\int_{\omega_{h}} \widetilde{\phi_{h i j, j}} u_{, i} d x\right| \text { with } \omega_{h}=\Omega-\Omega_{h},
$$

where $\tilde{\Phi}_{h}=\left(\tilde{\phi}_{h i j}\right)_{i, j=1,2} \in \tilde{\mathbf{V}}_{h}$ is a natural extension to $\bar{\Omega}$ of $\Phi_{h} \in \mathbf{V}_{h}$ defined in (3.21).

- Estimate for the first term on the right-hand side of (4.42)

$$
\left|\int_{\omega_{h}} A_{i j k l} \psi_{i j} \widetilde{\phi_{h k l}} \mathrm{~d} x\right| \leq \sum_{i, j, k, l=1}^{2}\left|\int_{\omega_{h}} A_{i j k l} \psi_{i j} \widetilde{\phi_{h k l}} \mathrm{~d} x\right|=\sum_{k, l=1}^{2}\left|\int_{\omega_{h}} u_{, k l} \widetilde{\phi_{h k l}} \mathrm{~d} x\right|,
$$

since $A_{i j k l} \psi_{i j}=A_{i j k l} a_{i j m n} u_{, m n}=\delta_{k m} \delta_{l n} u_{, m n}=u_{, k l}[$ see $(2.15)]$.

Then, since $u \in H^{3}(\Omega), \widetilde{\phi_{h k l}} \in H^{1}(\Omega)$, we can use (4.21).

Hence, for fixed $k, l=1,2$

$$
\begin{aligned}
& \mid \int_{\omega_{h}} u, k l \widetilde{\phi_{h k l}} \mathrm{~d} \Omega \mid \\
& \leq\left\|u u_{, k l}\right\|_{0, \omega_{h}}\left\|\widetilde{\phi_{h k l}}\right\|_{0, \omega_{h}} \\
& \leq\left(C h\left\|u_{, k l}\right\|_{1, \Omega}\right)\left(C h\left\|\widetilde{\phi_{h k l}}\right\|_{1, \Omega}\right) \leq C h^{2}\|u\|_{3, \Omega}\left\|\widetilde{\phi_{h k l}}\right\|_{1, \Omega} \leq C h^{2}\|u\|_{3, \Omega}\left\|\tilde{\Phi}_{h}\right\|_{1, \Omega} \\
& \Longrightarrow\left|\int_{\omega_{h}} A_{i j k l} \psi_{i j} \widetilde{\phi_{h k l}} \mathrm{~d} x\right| \leq C h^{2}\|u\|_{3, \Omega}\left\|\Phi_{h}\right\|_{1, \Omega_{h}} .
\end{aligned}
$$

\section{- Estimate for the second term on the right-hand side of (4.42)}

For fixed $i, j=1,2$,

$$
\left|\int_{\omega_{h}} \widetilde{\phi_{h i j, j}} u_{, i} \mathrm{~d} x\right| \leq\left(\operatorname{meas} \omega_{h}\right)^{\frac{1}{2}}\left\|u_{, i} \widetilde{\phi_{h i j, j}}\right\|_{0, \omega_{h}} \leq C h\left\|u_{, i} \widetilde{\phi_{h i j, j}}\right\|_{0, \omega_{h}}
$$

[since $u_{, i} \in H^{2}(\Omega) \hookrightarrow C^{0}(\bar{\Omega}), \widetilde{\phi_{h i j, j}} \in L^{2}(\Omega) \Longrightarrow u_{, i} \widetilde{\phi_{h i j, j}} \in L^{2}(\Omega)$ and $\operatorname{meas}\left(\omega_{h}\right)=O\left(h^{2}\right)($ see $(4.24))$ ].

But for fixed $i, j=1,2$

$$
\begin{aligned}
\left\|u_{, i} \widetilde{\phi_{h i j, j}}\right\|_{0, \omega_{h}}^{2} & \leq\left(\max _{x \in \bar{\Omega}}\left|u_{, i}(x)\right|\right)^{2}\left|\widetilde{\phi_{h i j, j}}\right|_{0, \omega_{h}}^{2} \leq\|u, i\|_{0, \infty, \Omega}^{2}\left|\widetilde{\phi_{h i j, j}}\right|_{0, \omega_{h}}^{2} \\
& \leq C\|u, i\|_{2, \Omega}^{2}\left\|\widetilde{\phi_{h i j}}\right\|_{1, \omega_{h}}^{2} \leq C\|u\|_{3, \Omega}^{2}\left\|\widetilde{\phi_{h i j}}\right\|_{1, \omega_{h}}^{2} \leq C h\|u\|_{3, \Omega}^{2}\left\|\phi_{h i j}\right\|_{1, \Omega_{h}}^{2},
\end{aligned}
$$


since the third inequality in (4.45) follows from $H^{2}(\Omega) \hookrightarrow L^{\infty}(\Omega)$ and the last inequality follows from (4.23). Then from (4.44) and (4.45),

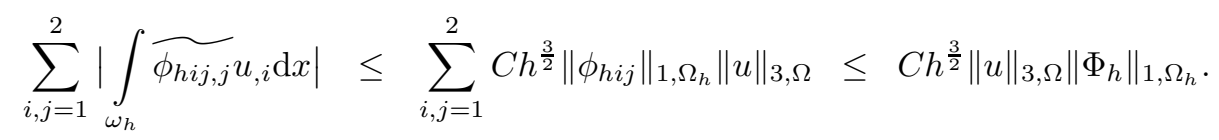

Finally, from $(4.42,4.43,4.46)$, we get the result (4.41).

Lemma 4.2. Let the quadrature scheme (3.11) with $i=2$, which is exact for $P_{2}(\hat{T})$, correspond to the definition (3.24) of $b_{h}^{N I}(\cdot, \cdot)$ and $\tilde{b}(\cdot, \cdot)$ be defined by $(3.16)$.

Then, $\forall \Phi \in \mathbf{V}\left(\Omega_{h}\right), \exists$ a tensor-valued function $\Theta_{h} \in \mathbf{V}_{h}$, for which the following hold:

$$
\tilde{b}\left(\Phi, \chi_{h}\right)=b_{h}^{N I}\left(\Theta_{h}, \chi_{h}\right) \forall \chi_{h} \in W_{h} \subset H_{0}^{1}\left(\Omega_{h}\right)
$$

and $\exists C>0$, independent of $h$, such that

$$
\left\|\Phi-\Theta_{h}\right\|_{r, \Omega_{h}} \leq C h^{1-r}\|\Phi\|_{1, \Omega},(r=0,1) .
$$

Proof. For $\phi \in H^{1}\left(\Omega_{h}\right)$, we can associate a $\phi_{h} \in \mathbf{X}_{h}$ such that

$$
\left\|\phi-\phi_{h}\right\|_{r, \Omega_{h}} \leq C h^{1-r}\|\phi\|_{1, \Omega_{h}}(r=0,1)
$$

for some $C>0$ independent of $h$.

$\Rightarrow \forall \Phi=\left(\phi_{i j}\right)_{i, j=1,2} \in \mathbf{V}\left(\Omega_{h}\right), \exists \Phi_{h} \in \mathbf{V}_{h}$ such that

$$
\begin{aligned}
&\left\|\Phi-\Phi_{h}\right\|_{r, \Omega_{h}}^{2}=\sum_{i, j=1}^{2}\left\|\phi_{i j}-\phi_{h i j}\right\|_{r, \Omega_{h}}^{2} \leq \sum_{i, j=1}^{2} C^{2} h^{2-2 r}\left\|\phi_{i j}\right\|_{1, \Omega_{h}}^{2}=C^{2} h^{2-2 r}\|\Phi\|_{1, \Omega_{h}}^{2}(r=0,1) \\
& \Rightarrow\left\|\Phi-\Phi_{h}\right\|_{r, \Omega_{h}} \leq C h^{1-r}\|\Phi\|_{1, \Omega_{h}} \quad(r=0,1) .
\end{aligned}
$$

Define an auxiliary bilinear form $\mathcal{B}_{h}(\cdot, \cdot): W_{h} \times W_{h} \longrightarrow \mathcal{R}$ by:

$$
\mathcal{B}_{h}\left(z_{h}, \mu_{h}\right)=\sum_{T \in \tau_{h}} \sum_{n=1}^{N_{2}} w_{n, T}^{2}\left(\nabla z_{h} \cdot \nabla \mu_{h}\right)\left(b_{n, T}^{2}\right) \quad \forall z_{h}, \mu_{h} \in W_{h}
$$

with $w_{n, T}^{2}>0,1 \leq n \leq N_{2}$, which corresponds to the quadrature scheme 3.11) with $i=2$ exact for $P_{2}(\hat{T})$, and a linear form $l_{h}(\cdot): W_{h} \longrightarrow \mathbb{R}$ by:

$$
l_{h}\left(\mu_{h}\right)=\tilde{b}\left(\Phi, \mu_{h}\right)-b_{h}^{N I}\left(\Phi_{h}, \mu_{h}\right) \quad \forall \mu_{h} \in W_{h}
$$

for fixed elements $\Phi \in \mathbf{V}\left(\Omega_{h}\right), \Phi_{h} \in \mathbf{V}_{h}$ satisfying (4.50).

$\mathcal{B}_{h}(\cdot, \cdot)$ is continuous on $W_{h} \times W_{h}$ and $W_{h}$-elliptic. 
In fact,

$$
\begin{aligned}
\mathcal{B}_{h}\left(\mu_{h}, \mu_{h}\right) & =\sum_{T \in \tau_{h}} \sum_{n=1}^{N_{2}} w_{n, T}^{2}\left(\nabla \mu_{h} \cdot \nabla \mu_{h}\right)\left(b_{n, T}^{2}\right) \\
& \geq C\left|\mu_{h}\right|_{1, \Omega_{h}}^{2}=C\left|\tilde{\mu_{h}}\right|_{1, \Omega}^{2} \quad\left(\text { since } \tilde{\mu}_{h}=0 \text { outside } \Omega_{h}\right) \\
& \geq C(\Omega)\left\|\tilde{\mu}_{h}\right\|_{1, \Omega}^{2} \quad(\text { by virtue of Friedrichs' inequality) } \\
& =C(\Omega)\left\|\mu_{h}\right\|_{1, \Omega_{h}}^{2} \quad\left(\text { since } \tilde{\mu}_{h}=\mu_{h} \text { in } \Omega_{h}, \tilde{\mu}_{h}=0 \text { outside } \Omega_{h}\right) \\
\Longrightarrow \mathcal{B}_{h}\left(\mu_{h}, \mu_{h}\right) & \geq C(\Omega)\left\|\mu_{h}\right\|_{1, \Omega_{h}}^{2} \quad \forall \mu_{h} \in W_{h} .
\end{aligned}
$$

$l_{h}(\cdot)$ is continuous on $W_{h}$.

Hence, from Lax-Milgram lemma, $\exists$ a unique $z_{h} \in W_{h}$ such that

$$
\mathcal{B}_{h}\left(z_{h}, \mu_{h}\right)=\tilde{b}\left(\Phi, \mu_{h}\right)-b_{h}^{N I}\left(\Phi_{h}, \mu_{h}\right) \forall \mu_{h} \in W_{h}
$$

for fixed $\Phi \in \mathbf{V}\left(\Omega_{h}\right)$ and $\Phi_{h} \in \mathbf{V}_{h}$ satisfying (4.50).

Choose $\underline{\sigma}_{h}=\left(z_{h} \delta_{i j}\right)_{i, j=1,2}$ with $z_{h} \in W_{h}$. Then

$$
\underline{\sigma}_{h} \in \mathbf{V}_{h} \text { with }\left\|\underline{\sigma}_{h}\right\|_{1, \Omega_{h}}=\sqrt{ } 2\left\|z_{h}\right\|_{1, \Omega_{h}}
$$

and

$$
\begin{aligned}
b_{h}^{N I}\left(\underline{\sigma}_{h}, \mu_{h}\right) & =\mathcal{B}_{h}\left(z_{h}, \mu_{h}\right)=\tilde{b}\left(\Phi, \mu_{h}\right)-b_{h}^{N I}\left(\Phi_{h}, \mu_{h}\right) & & {[\operatorname{using}(4.54)] } \\
\Longrightarrow b_{h}^{N I}\left(\underline{\sigma}_{h}, \mu_{h}\right)+b_{h}^{N I}\left(\Phi_{h}, \mu_{h}\right) & =b_{h}^{N I}\left(\underline{\sigma}_{h}+\Phi_{h}, \mu_{h}\right)=\tilde{b}\left(\Phi, \mu_{h}\right) & & \forall \mu_{h} \in W_{h} \\
\Longrightarrow \text { the result }(4.47) \text { holds with } \Theta_{h} & =\left(\underline{\sigma}_{h}+\Phi_{h}\right) \in \mathbf{V}_{h}, \Phi_{h} \text { satisfying (4.50). } & &
\end{aligned}
$$

- Estimate for $\left\|\Phi-\Theta_{h}\right\|_{1, \Omega_{h}}$

$\forall$ fixed elements $\Phi \in \mathbf{V}\left(\Omega_{h}\right), \Phi_{h} \in \mathbf{V}_{h}$ satisfying $(4.50)$, we get from $(4.53,4.54)$ and the continuity of $l_{h}(\cdot)$ :

$$
\begin{aligned}
C\left\|z_{h}\right\|_{1, \Omega_{h}}^{2} & \leq \mathcal{B}_{h}\left(z_{h}, z_{h}\right) \leq M\left\|z_{h}\right\|_{1, \Omega_{h}}\left(\|\Phi\|_{1, \Omega_{h}}+\left\|\Phi_{h}\right\|_{1, \Omega_{h}}\right) \\
& \leq M\left\|z_{h}\right\|_{1, \Omega_{h}}\left(2\|\Phi\|_{1, \Omega_{h}}+\left\|\Phi-\Phi_{h}\right\|_{1, \Omega_{h}}\right) \\
& \leq C M\left\|z_{h}\right\|_{1, \Omega_{h}}\|\Phi\|_{1, \Omega_{h}}[\text { by virtue of }(4.50)] \\
\Longrightarrow\left\|z_{h}\right\|_{1, \Omega_{h}} & \leq C\|\Phi\|_{1, \Omega_{h}} .
\end{aligned}
$$

Hence from (4.55) and (4.57), and the definition of $\Theta_{h}$, we have

$$
\begin{aligned}
\left\|\underline{\sigma}_{h}\right\|_{1, \Omega_{h}} & =\sqrt{ } 2\left\|z_{h}\right\|_{1, \Omega_{h}} \leq C\|\Phi\|_{1, \Omega_{h}} . \\
\Longrightarrow\left\|\Theta_{h}-\Phi_{h}\right\|_{1, \Omega_{h}} & \leq C\|\Phi\|_{1, \Omega_{h}} . \\
\Longrightarrow\left\|\Phi-\Theta_{h}\right\|_{1, \Omega_{h}} & \leq\left\|\Phi-\Phi_{h}\right\|_{1, \Omega_{h}}+\left\|\Phi_{h}-\Theta_{h}\right\|_{1, \Omega_{h}} \\
& \leq C\|\Phi\|_{1, \Omega_{h}} \leq C\|\Phi\|_{1, \Omega} .
\end{aligned}
$$

- Estimate for $\left\|\Phi-\Theta_{h}\right\|_{0, \Omega_{h}}$

Since $\Omega$ is convex, $\forall g \in L^{2}(\Omega)$, define $\chi \in H^{2}(\Omega) \cap H_{0}^{1}(\Omega)$ as the unique solution of:

$$
-\Delta \chi=g \text { in } \Omega,\left.\quad \chi\right|_{\Gamma}=0 \quad \text { with }\|\chi\|_{2, \Omega} \leq C\|g\|_{0, \Omega} .
$$


$\forall z_{h} \in W_{h}$ with $\tilde{z_{h}} \in \tilde{W}_{h}$, we have

$$
\left\|z_{h}\right\|_{0, \Omega_{h}}=\left\|\tilde{z}_{h}\right\|_{0, \Omega}=\sup _{g \in L^{2}(\Omega)} \frac{\left|\int_{\Omega} \tilde{z}_{h} g \mathrm{~d} \Omega\right|}{\|g\|_{0, \Omega}}
$$

Then from (4.61),

$$
\begin{gathered}
-\int_{\Omega}(\Delta \chi) \cdot \tilde{z}_{h} \mathrm{~d} \Omega=\int_{\Omega} g \tilde{z}_{h} \mathrm{~d} \Omega \quad \forall \tilde{z}_{h} \in \tilde{W}_{h} \subset H_{0}^{1}(\Omega) \\
\Longrightarrow \int_{\Omega_{h}}(\nabla \chi) \cdot \nabla z_{h} \mathrm{~d} \Omega_{h}=\int_{\Omega_{h}} g z_{h} \mathrm{~d} \Omega_{h} \quad \forall z_{h} \in W_{h}, \text { since } \tilde{z}_{h}=0 \text { in } \Omega-\Omega_{h} .
\end{gathered}
$$

Hence, using (4.54), we get:

$$
\begin{aligned}
\left|\int_{\Omega_{h}} g z_{h} \mathrm{~d} \Omega_{h}\right|=\left|\int_{\Omega_{h}} \nabla \chi \cdot \nabla z_{h} \mathrm{~d} \Omega_{h}\right| \leq & \left|\int_{\Omega_{h}} \nabla\left(\chi-\chi_{h}\right) . \nabla z_{h} \mathrm{~d} \Omega_{h}\right|+\mid \int_{\Omega_{h}} \nabla \chi_{h} . \nabla z_{h} \mathrm{~d} \Omega_{h} \\
& -\sum_{T \in \tau_{h}} \sum_{n=1}^{N_{2}} w_{n, T}^{2}\left(\nabla \chi_{h} . \nabla z_{h}\right)\left(b_{n, T}^{2}\right)|+| \tilde{b}\left(\Phi, \chi_{h}\right)-b_{h}^{N I}\left(\Phi_{h}, \chi_{h}\right) \mid
\end{aligned}
$$

$\forall \chi_{h} \in W_{h}$ and for fixed elements $\Phi \in \mathbf{V}\left(\Omega_{h}\right), \Phi_{h} \in \mathbf{V}_{h}$ satisfying (4.50).

Then, for $\chi_{h}=\mathcal{P}_{0 h} \chi \in W_{h}$ with $\chi \in H^{2}(\Omega) \cap H_{0}^{1}(\Omega)$ defined in (4.4), we have:

$$
\int_{\Omega_{h}} \nabla\left(\mathcal{P}_{0 h} \chi\right) \cdot \nabla z_{h} \mathrm{~d} \Omega_{h}=\sum_{T \in \tau_{h}} \sum_{n=1}^{N_{2}} w_{n, T}^{2}\left(\left(\nabla \mathcal{P}_{0 h} \chi\right) \cdot \nabla z_{h}\right)\left(b_{n, T}^{2}\right),
$$

and consequently,

$$
\begin{aligned}
\left|\int_{\Omega_{h}} g z_{h} \mathrm{~d} \Omega_{h}\right| \leq & \left|\int_{\Omega_{h}} \nabla\left(\chi-\mathcal{P}_{0 h} \chi\right) \cdot \nabla z_{h} \mathrm{~d} \Omega_{h}\right|+\left|\tilde{b}\left(\Phi-\Phi_{h}, \mathcal{P}_{0 h} \chi-\chi\right)\right| \\
& +\left|\tilde{b}\left(\Phi-\Phi_{h}, \chi\right)-b\left(\Phi-\tilde{\Phi_{h}}, \chi\right)\right|+\left|b\left(\Phi-\tilde{\Phi_{h}}, \chi\right)\right| .
\end{aligned}
$$

- Estimate for the first term on the right-hand side of (4.64)

Using (4.5) and (4.57),

$$
\begin{aligned}
\left|\int_{\Omega_{h}} \nabla\left(\chi-\mathcal{P}_{0 h} \chi\right) \cdot \nabla z_{h} \mathrm{~d} \Omega_{h}\right| & \leq\left|\chi-\mathcal{P}_{0 h} \chi\right|_{1, \Omega_{h}}\left|z_{h}\right|_{1, \Omega_{h}} \leq C h\|g\|_{0, \Omega}\left\|z_{h}\right\|_{1, \Omega_{h}} \\
& \leq C h\|g\|_{0, \Omega}\|\Phi\|_{1, \Omega_{h}} \quad[\text { by (4.61)]. }
\end{aligned}
$$

- Estimate for the second term on the right-hand side of (4.64)

Using the continuity of $\tilde{b}(\cdot, \cdot),(4.5,4.50)$ and $(4.61)$, we have

$$
\left|\tilde{b}\left(\Phi-\Phi_{h}, \mathcal{P}_{0 h} \chi-\chi\right)\right| \leq \tilde{m}\left\|\Phi-\Phi_{h}\right\|_{1, \Omega_{h}}\left\|\mathcal{P}_{0 h} \chi-\chi\right\|_{1, \Omega_{h}} \leq C h\|\Phi\|_{1, \Omega_{h}}\|g\|_{0, \Omega} .
$$


- Estimate for the third term on the right-hand side of (4.64)

$$
\begin{aligned}
\left|\tilde{b}\left(\Phi-\Phi_{h}, \chi\right)-b\left(\Phi-\tilde{\Phi_{h}}, \chi\right)\right| & =\left|\int_{\Omega_{h}}\left(\phi_{i j}-\phi_{h i j}\right)_{, j} \chi, i \mathrm{~d} \Omega_{h}-\int_{\Omega}\left(\phi_{i j}-\tilde{\phi}_{h i j}\right)_{, j} \chi_{, i} \mathrm{~d} \Omega\right| \\
& \leq\left|\int_{\omega_{h}}\left(\phi_{i j}-\tilde{\phi}_{h i j}\right)_{, j} \chi, i \mathrm{~d} x\right| \leq\left\|\left(\phi_{i j}-\tilde{\phi}_{h i j}\right)_{, j}\right\|_{0, \omega_{h}}\|\chi, i\|_{0, \omega_{h}} \\
& \leq C h\|\chi\|_{2, \Omega} \sum_{i, j=1}^{2}\left\|\left(\phi_{i j}-\tilde{\phi}_{h i j}\right)_{, j}\right\|_{0, \omega_{h}} \quad[\operatorname{using}(4.21)] .
\end{aligned}
$$

For fixed $i, j=1,2\left\|\left(\phi_{i j}-\tilde{\phi}_{h i j}\right)_{, j}\right\|_{0, \omega_{h}} \leq\left\|\phi_{i j}-\tilde{\phi}_{h i j}\right\|_{1, \omega_{h}} \leq\left\|\phi_{i j}\right\|_{1, \omega_{h}}+\left\|\tilde{\phi}_{h i j}\right\|_{1, \omega_{h}}$.

From (4.23), $\forall i, j=1,2, \tilde{\phi}_{h i j} \in \tilde{X}_{h}$ defined in (3.20),

$$
\left\|\tilde{\phi}_{h i j}\right\|_{1, \omega_{h}} \leq C h^{1 / 2}\left\|\phi_{h i j}\right\|_{1, \Omega_{h}} \leq C h^{1 / 2}\left\|\Phi_{h}\right\|_{1, \Omega_{h}} .
$$

Then, using (4.50),

$$
\left\|\tilde{\phi}_{h i j}\right\|_{1, \omega_{h}} \leq C h^{1 / 2}\left(\left\|\Phi-\Phi_{h}\right\|_{1, \Omega_{h}}+\|\Phi\|_{1, \Omega_{h}}\right) \leq C h^{1 / 2}\left(\|\Phi\|_{1, \Omega_{h}}+\|\Phi\|_{1, \Omega_{h}}\right) \leq C h^{1 / 2}\|\Phi\|_{1, \Omega} .
$$

From (4.67-4.69), we have

$$
\sum_{i, j=1}^{2}\left\|\left(\phi_{i j}-\tilde{\phi}_{h i j}\right)_{, j}\right\|_{0, \omega_{h}} \leq\|\Phi\|_{1, \Omega}+C h^{1 / 2}\|\Phi\|_{1, \Omega} \leq C\|\Phi\|_{1, \Omega}
$$

Hence, from (4.67),

$$
\left|\tilde{b}\left(\Phi-\Phi_{h}, \chi\right)-b\left(\Phi-\tilde{\Phi_{h}}, \chi\right)\right| \leq C h\|\chi\|_{2, \Omega}\|\Phi\|_{1, \Omega} \leq C h\|g\|_{0, \Omega}\|\Phi\|_{1, \Omega} .
$$

- Estimate for the fourth term on the right-hand side of (4.64)

For $\chi \in H^{2}(\Omega) \cap H_{0}^{1}(\Omega)$,

$$
\left|b\left(\Phi-\tilde{\Phi}_{h}, \chi\right)\right| \leq C\left\|\Phi-\tilde{\Phi}_{h}\right\|_{0, \Omega}|\chi|_{2, \Omega}
$$

with

$$
\left\|\Phi-\tilde{\Phi}_{h}\right\|_{0, \Omega}^{2}=\left\|\Phi-\Phi_{h}\right\|_{0, \Omega_{h}}^{2}+\left\|\Phi-\Phi_{h}\right\|_{0, \omega_{h}}^{2} \quad\left(\omega_{h}=\Omega-\Omega_{h}\right) .
$$


For fixed $i, j=1,2,\left(\phi_{i j}-\tilde{\phi}_{h i j}\right) \in H^{1}(\Omega)$ and hence from (4.21),

$$
\left\|\phi_{i j}-\tilde{\phi}_{h i j}\right\|_{0, \omega_{h}}^{2} \leq C^{2} h^{2}\left\|\phi_{i j}-\tilde{\phi}_{h i j}\right\|_{1, \Omega}^{2} .
$$

But

$$
\begin{aligned}
\left\|\phi_{i j}-\tilde{\phi}_{h i j}\right\|_{1, \Omega}^{2} & =\left\|\phi_{i j}-\phi_{h i j}\right\|_{1, \Omega_{h}}^{2}+\left\|\phi_{i j}-\tilde{\phi}_{h i j}\right\|_{1, \omega_{h}}^{2} \\
& \leq C\left\|\phi_{i j}\right\|_{1, \Omega_{h}}^{2}+\left(\left\|\phi_{i j}\right\|_{1, \omega_{h}}+\left\|\tilde{\phi}_{h i j}\right\|_{1, \omega_{h}}\right)^{2} \leq C\left[\left\|\phi_{i j}\right\|_{1, \Omega}^{2}+\left\|\phi_{i j}\right\|_{1, \Omega}^{2}+\left\|\tilde{\phi}_{h i j}\right\|_{1, \omega_{h}}^{2}\right] \\
\Longrightarrow\left\|\phi_{i j}-\tilde{\phi}_{h i j}\right\|_{1, \Omega}^{2} & \leq C\left[\|\Phi\|_{1, \Omega}^{2}+C h\|\Phi\|_{1, \Omega}^{2}\right][\operatorname{using}(4.23)] \\
\Longrightarrow \sum_{i, j=1}^{2}\left\|\phi_{i j}-\tilde{\phi}_{h i j}\right\|_{1, \Omega}^{2} & \leq C\|\Phi\|_{1, \Omega}^{2} \Longrightarrow\left\|\Phi-\tilde{\Phi}_{h}\right\|_{1, \Omega} \leq C\|\Phi\|_{1, \Omega} .
\end{aligned}
$$

Hence

$$
\left\|\Phi-\tilde{\Phi}_{h}\right\|_{0, \omega_{h}} \leq C h\|\Phi\|_{1, \Omega} \quad[\text { from (4.74)] }
$$

Then from (4.73)

$$
\left\|\Phi-\tilde{\Phi}_{h}\right\|_{0, \Omega}^{2} \leq C h^{2}\|\Phi\|_{1, \Omega}^{2}+C^{2} h^{2}\|\Phi\|_{1, \Omega}^{2} \leq C^{2} h^{2}\|\Phi\|_{1, \Omega}^{2} .
$$

Hence,

$$
\left|b\left(\Phi-\tilde{\Phi}_{h}, \chi\right)\right| \leq C h\|\Phi\|_{1, \Omega}\|\chi\|_{2, \Omega} \leq C h\|g\|_{0, \Omega}\|\Phi\|_{1, \Omega} \text { [using (4.61)]. }
$$

Substituting the estimates $(4.65-4.66,4.71,4.75)$ in (4.64) and using the result $\|\Phi\|_{1, \Omega_{h}} \leq\|\Phi\|_{1, \Omega}$, we have

$$
\begin{aligned}
\left|\int_{\Omega} g \tilde{z}_{h} \mathrm{~d} \Omega\right| & =\left|\int_{\Omega_{h}} g z_{h} \mathrm{~d} \Omega_{h}\right| \leq C h\|g\|_{0, \Omega}\left(\|\Phi\|_{1, \Omega}+\|\Phi\|_{1, \Omega}+\|\Phi\|_{1, \Omega}+\|\Phi\|_{1, \Omega}\right) \\
& \leq C h\|g\|_{0, \Omega}\|\Phi\|_{1, \Omega} .
\end{aligned}
$$

Then, from $(4.62,4.76)$, we have

$$
\left\|z_{h}\right\|_{0, \Omega_{h}}=\left\|\tilde{z}_{h}\right\|_{0, \Omega}=\sup _{g \in L^{2}(\Omega)} \frac{\left|\int_{\Omega} \tilde{z}_{h} g \mathrm{~d} \Omega\right|}{\|g\|_{0, \Omega}} \leq C h\|\Phi\|_{1, \Omega} .
$$

Since, $\underline{\sigma}_{h}=\left(z_{h} \delta_{i j}\right)$ with $z_{h} \in W_{h}$ and $\underline{\Theta}_{h}=\underline{\sigma}_{h}+\Phi_{h} \in \mathbf{V}_{h}$, [see (4.56)], we have

$$
\left\|\Theta_{h}-\Phi_{h}\right\|_{0, \Omega_{h}}=\left\|\underline{\sigma}_{h}\right\|_{0, \Omega_{h}}=\sqrt{ } 2\left\|z_{h}\right\|_{0, \Omega_{h}} \leq C h\|\Phi\|_{1, \Omega} .
$$

Hence

$$
\begin{aligned}
\left\|\Phi-\Theta_{h}\right\|_{0, \Omega_{h}} & \leq\left\|\Phi-\Phi_{h}\right\|_{0, \Omega_{h}}+\left\|\Phi_{h}-\Theta_{h}\right\|_{0, \Omega_{h}} \\
& \leq C h\|\Phi\|_{1, \Omega_{h}}+C h\|\Phi\|_{1, \Omega} \quad[\text { from (4.50) and (4.77)] } \\
\Longrightarrow\left\|\Phi-\Theta_{h}\right\|_{0, \Omega_{h}} & \leq C h\|\Phi\|_{1, \Omega} .
\end{aligned}
$$


Thus, (4.60) and (4.78) establish the result (4.48).

\section{Theorem 4.1.}

- Suppose that the assumptions of Theorem 2.1, Propositions 4.3 and 4.4 hold.

- Let $\left\{\tau_{h}\right\}$ (resp. $\left\{\tau_{h}^{\text {exact }}\right\}$ ) be a family of quasi-uniform, regular, admissible triangulations [17] of $\bar{\Omega}_{h}=\Omega_{h} \cup \Gamma_{h}$ (resp. $\bar{\Omega}=\Omega \cup \Gamma$ ) defined in (3.3) with $\left.0<h<h_{0}, h_{0} \in\right] 0,1[$.

- Let the quadrature scheme (3.11) with $i=1$ [resp. $i=2]$, which is exact for $P_{4}(\hat{T})$ [resp. $\left.P_{2}(\hat{T})\right]$ correspond to the definition (3.23) of $A_{h}^{N I}(\cdot, \cdot)$ [resp. (3.24) of $\left.b_{h}^{N I}(\cdot, \cdot)\right]$.

Then, $\exists C>0$, independent of $h$, such that

$$
\begin{gathered}
\left\|\Psi-\Psi_{h}\right\|_{0, \Omega_{h}} \leq C \sqrt{h}\left[\|u\|_{3, \Omega}+h^{1 / 2}\|\Psi\|_{1, \Omega}\right] \\
\left\|u-u_{h}\right\|_{1, \Omega_{h}} \leq C \sqrt{h}\left[\|u\|_{3, \Omega}+h^{1 / 2}\|\Psi\|_{1, \Omega}\right]
\end{gathered}
$$

where $(\Psi, u) \in \mathbf{V} \times W$ [resp. $\left.\left(\Psi_{h}, u_{h}\right) \in \mathbf{V}_{h} \times W_{h}\right]$ is the unique solution of $(\mathbf{Q})\left[\right.$ resp. $\left.\left(\mathbf{Q}_{\mathbf{h}}\right)\right]$.

Proof. Since $\Psi \downarrow_{\Omega_{h}} \in \mathbf{V}\left(\Omega_{h}\right)$, from Lemma $4.2, \exists \Theta_{h} \in \mathbf{V}_{h}$ such that

$$
b_{h}^{N I}\left(\Theta_{h}, \chi_{h}\right)=\tilde{b}\left(\Psi, \chi_{h}\right)=\int_{\Omega_{h}} \psi_{i j, j} \chi_{h, i} \mathrm{~d} \Omega_{h} \quad \forall \chi_{h} \in W_{h}
$$

and

$$
\left\|\Psi-\Theta_{h}\right\|_{r, \Omega_{h}} \leq C h^{1-r}\|\Psi\|_{1, \Omega} \quad(r=0,1) .
$$

Then, from (4.81), the definition of $\tilde{W}_{h}$, and the second equation of (2.10),

$$
b_{h}^{N I}\left(\Theta_{h}, \chi_{h}\right)=\int_{\Omega} \psi_{i j, j} \tilde{\chi}_{h, i} \mathrm{~d} \Omega=b\left(\Psi, \tilde{\chi}_{h}\right)=-\left\langle f, \tilde{\chi}_{h}\right\rangle_{0, \Omega}=-\left\langle f, \chi_{h}\right\rangle_{0, \Omega_{h}} \quad \forall \chi_{h} \in W_{h} \text { with } \tilde{\chi}_{h} \in \tilde{W}_{h} .
$$

Hence using the second equation of (3.25) and (4.83), we have

$$
b_{h}^{N I}\left(\Psi_{h}-\Theta_{h}, \chi_{h}\right)=-\left\langle f, \chi_{h}\right\rangle_{0, \Omega_{h}}+\left\langle f, \chi_{h}\right\rangle_{0, \Omega_{h}}=0 \quad \forall \chi_{h} \in W_{h} .
$$


From the ellipticity of $A_{h}^{N I}(\cdot, \cdot)$ in (3.27), we have for $\Theta_{h} \in \mathbf{V}_{h}$ corresponding to $\Psi \in \mathbf{V}\left(\Omega_{h}\right)$ satisfying $(4.81-4.83)$,

$$
\begin{aligned}
\alpha\left\|\Psi_{h}-\Theta_{h}\right\|_{0, \Omega_{h}}^{2} \leq A_{h}^{N I}\left(\Psi_{h}-\Theta_{h}, \Psi_{h}-\Theta_{h}\right)= & {\left[\tilde{A}\left(\Psi-\Theta_{h}, \Psi_{h}-\Theta_{h}\right)-\tilde{A}\left(\Psi, \Psi_{h}-\Theta_{h}\right)\right] } \\
& +\left[\tilde{A}\left(\Theta_{h}, \Psi_{h}-\Theta_{h}\right)-A_{h}^{N I}\left(\Theta_{h}, \Psi_{h}-\Theta_{h}\right)\right] \\
& -b_{h}^{N I}\left(\Psi_{h}-\Theta_{h}, u_{h}\right) \quad[\text { using the first equation of }(3.25)] \\
= & \tilde{A}\left(\Psi-\Theta_{h}, \Psi_{h}-\Theta_{h}\right)-\left[\tilde{A}\left(\Psi, \Psi_{h}-\Theta_{h}\right)+\tilde{b}\left(\Psi_{h}-\Theta_{h}, u\right)\right] \\
& +\tilde{b}\left(\Psi_{h}-\Theta_{h}, u\right)+\left[\tilde{A}\left(\Theta_{h}, \Psi_{h}-\Theta_{h}\right)-A_{h}^{N I}\left(\Theta_{h}, \Psi_{h}-\Theta_{h}\right)\right] \\
& -b_{h}^{N I}\left(\Psi_{h}-\Theta_{h}, u_{h}\right) \\
= & \tilde{A}\left(\Psi-\Theta_{h}, \Psi_{h}-\Theta_{h}\right)+\tilde{b}\left(\Psi_{h}-\Theta_{h}, u-\mathcal{P}_{0 h} u\right) \\
& -\left[\tilde{A}\left(\Psi, \Psi_{h}-\Theta_{h}\right)+\tilde{b}\left(\Psi_{h}-\Theta_{h}, u\right)\right] \\
& +\left[\tilde{A}\left(\Theta_{h}, \Psi_{h}-\Theta_{h}\right)-A_{h}^{N I}\left(\Theta_{h}, \Psi_{h}-\Theta_{h}\right)\right] \\
& +\left[\tilde{b}\left(\Psi_{h}-\Theta_{h}, \mathcal{P}_{0 h} u\right)-b_{h}^{N I}\left(\Psi_{h}-\Theta_{h}, \mathcal{P}_{0 h} u\right)\right],
\end{aligned}
$$

which has been obtained by using (4.84) and the definition (4.4) of $\mathcal{P}_{0 h} u \in W_{h}$. Since the quadrature scheme (3.11) with $i=2$ corresponding to the definition of $b_{h}^{N I}(\cdot, \cdot)$ is exact for $P_{2}(\hat{T})$,

$$
\tilde{b}\left(\Psi_{h}-\Theta_{h}, \mathcal{P}_{0 h} u\right)-b_{h}^{N I}\left(\Psi_{h}-\Theta_{h}, \mathcal{P}_{0 h} u\right)=0 .
$$

Hence, applying the triangular inequality, the continuity of $\tilde{A}(\cdot, \cdot)$ and $\tilde{b}(\cdot, \cdot)$ and finally dividing both sides by $\alpha\left\|\Psi_{h}-\Theta_{h}\right\|_{0, \Omega_{h}}$, we get from (4.85-4.86):

$$
\begin{aligned}
\left\|\Psi_{h}-\Theta_{h}\right\|_{0, \Omega_{h}} \leq & C\left\{\left[\frac{\tilde{M}\left\|\Psi-\Theta_{h}\right\|_{0, \Omega_{h}}\left\|\Psi_{h}-\Theta_{h}\right\|_{0, \Omega_{h}}}{\left\|\Psi_{h}-\Theta_{h}\right\|_{0, \Omega_{h}}}+\frac{\tilde{m}\left\|\Psi_{h}-\Theta_{h}\right\|_{1, \Omega_{h}}\left\|u-\mathcal{P}_{0 h} u\right\|_{1, \Omega_{h}}}{\left\|\Psi_{h}-\Theta_{h}\right\|_{0, \Omega_{h}}}\right]\right. \\
& \left.+\left[\frac{\tilde{A}\left(\Psi, \Psi_{h}-\Theta_{h}\right)+\tilde{b}\left(\Psi_{h}-\Theta_{h}, u\right) \mid}{\left\|\Psi_{h}-\Theta_{h}\right\|_{0, \Omega_{h}}}\right]+\left[\frac{\left|\tilde{A}\left(\Theta_{h}, \Psi_{h}-\Theta_{h}\right)-A_{h}^{N I}\left(\Theta_{h}, \Psi_{h}-\Theta_{h}\right)\right|}{\left\|\Psi_{h}-\Theta_{h}\right\|_{0, \Omega_{h}}}\right]\right\} .
\end{aligned}
$$

Using $\left\|\Psi-\Theta_{h}\right\|_{0, \Omega_{h}} \leq C h\|\Psi\|_{1, \Omega}[$ from (4.82)]

$$
\begin{aligned}
& \left\|u-\mathcal{P}_{0 h} u\right\|_{1, \Omega_{h}} \leq C h^{3 / 2}\|u\|_{3, \Omega} \text { for } u \in H^{3}(\Omega) \cap H_{0}^{2}(\Omega),[\text { from (4.6)], } \\
& \left\|\Psi_{h}-\Theta_{h}\right\|_{1, \Omega_{h}} \leq \frac{C}{h}\left\|\Psi_{h}-\Theta_{h}\right\|_{0, \Omega_{h}},[\text { from }(4.20)],
\end{aligned}
$$

and [from Propositions 4.3 and 4.4],

$$
\begin{aligned}
\left|\tilde{A}\left(\Theta_{h}, \Psi_{h}-\Theta_{h}\right)-A_{h}^{N I}\left(\Theta_{h}, \Psi_{h}-\Theta_{h}\right)\right| & \leq C h\|A\|_{1, \infty, \Omega}\left\|\Theta_{h}\right\|_{0, \Omega_{h}}\left\|\Psi_{h}-\Theta_{h}\right\|_{0, \Omega_{h}} \\
\left|\tilde{A}\left(\Psi, \Psi_{h}-\Theta_{h}\right)+\tilde{b}\left(\Psi_{h}-\Theta_{h}, u\right)\right| & \leq C h^{3 / 2}(1+\sqrt{h})\|u\|_{3, \Omega}\left\|\Psi_{h}-\Theta_{h}\right\|_{1, \Omega_{h}},
\end{aligned}
$$


we have

$$
\left\|\Psi_{h}-\Theta_{h}\right\|_{0, \Omega_{h}} \leq C\left[h\|\Psi\|_{1, \Omega}+\sqrt{h}\|u\|_{3, \Omega}+\sqrt{h}(1+\sqrt{h})\|u\|_{3, \Omega}+h\|A\|_{1, \infty, \Omega}\|\Theta\|_{0, \Omega_{h}}\right]
$$

with $C>0$, independent of $h$.

Since $\left\|\Theta_{h}\right\|_{0, \Omega_{h}} \leq\left\|\Psi-\Theta_{h}\right\|_{0, \Omega_{h}}+\|\Psi\|_{0, \Omega_{h}} \leq C h\|\Psi\|_{1, \Omega}+\|\Psi\|_{1, \Omega} \leq C\|\Psi\|_{1, \Omega}$, we get from (4.91):

$$
\left\|\Psi_{h}-\Theta_{h}\right\|_{0, \Omega h} \leq C\left[h^{1 / 2}(2+\sqrt{h})\|u\|_{3, \Omega}+h\|\Psi\|_{1, \Omega}\right] \leq C h^{1 / 2}\left[\|u\|_{3, \Omega}+h^{1 / 2}\|\Psi\|_{1, \Omega}\right] .
$$

Hence

$$
\begin{aligned}
\left\|\Psi-\Psi_{h}\right\|_{0, \Omega_{h}} & \leq\left\|\Psi-\Theta_{h}\right\|_{0, \Omega_{h}}+\left\|\Psi_{h}-\Theta_{h}\right\|_{0, \Omega_{h}} \\
& \leq C\left[h\|\Psi\|_{1, \Omega}+h^{1 / 2}\left(\|u\|_{3, \Omega}+h^{1 / 2}\|\Psi\|_{1, \Omega}\right)\right] \\
\Longrightarrow\left\|\Psi-\Psi_{h}\right\|_{0, \Omega_{h}} & \leq C h^{1 / 2}\left[\|u\|_{3, \Omega}+h^{1 / 2}\|\Psi\|_{1, \Omega}\right] \text { with } C>0 \text {, independent of } h .
\end{aligned}
$$

Now, we will prove (4.80).

- Estimate for $\left\|u-u_{h}\right\|_{1, \Omega_{h}}$

From the discrete Brezzi-Babuška condition (3.28) for $b_{h}^{N I}(\cdot, \cdot)$, we have

$$
\beta_{1}\left\|u_{h}-\mathcal{P}_{0 h} u\right\|_{1, \Omega_{h}} \leq \sup _{\Phi_{h} \in \mathbf{V}_{h}-\{0\}} \frac{\left|b_{h}^{N I}\left(\Phi_{h}, u_{h}-\mathcal{P}_{0 h} u\right)\right|}{\left\|\Phi_{h}\right\|_{1, \Omega_{h}}}
$$

But

$$
\begin{aligned}
b_{h}^{N I}\left(\Phi_{h}, u_{h}-\mathcal{P}_{0 h} u\right)= & \tilde{b}\left(\Phi_{h}, u-\mathcal{P}_{0 h} u\right)+b_{h}^{N I}\left(\Phi_{h}, u_{h}\right)-\tilde{b}\left(\Phi_{h}, u\right)+\left[\tilde{b}\left(\Phi_{h}, \mathcal{P}_{0 h} u\right)-b_{h}^{N I}\left(\Phi_{h}, \mathcal{P}_{0 h} u\right)\right] \\
& +\tilde{A}\left(\Psi, \Phi_{h}\right)-\tilde{A}\left(\Psi, \Phi_{h}\right)
\end{aligned}
$$

where

$$
\begin{aligned}
& \tilde{b}\left(\Phi_{h}, \mathcal{P}_{0 h} u\right)-b_{h}^{N I}\left(\Phi_{h}, \mathcal{P}_{0 h} u\right)=0 \quad \forall \Phi_{h} \in \mathbf{V}_{h} \\
& \Longrightarrow b_{h}^{N I}\left(\Phi_{h}, u_{h}-\mathcal{P}_{0 h} u\right)=\quad \tilde{b}\left(\Phi_{h}, u-\mathcal{P}_{0 h} u\right)-A_{h}^{N I}\left(\Psi_{h}, \Phi_{h}\right)-\left[\tilde{A}\left(\Psi, \Phi_{h}\right)+\tilde{b}\left(\Phi_{h}, u\right)\right] \\
&+\tilde{A}\left(\Psi-\Psi_{h}, \Phi_{h}\right)+\tilde{A}\left(\Psi_{h}, \Phi_{h}\right)[\operatorname{using}(3.25)] \\
& \Longrightarrow b_{h}^{N I}\left(\Phi_{h}, u_{h}-\mathcal{P}_{0 h} u\right)= \tilde{A}\left(\Psi-\Psi_{h}, \Phi_{h}\right)+\tilde{b}\left(\Phi_{h}, u-\mathcal{P}_{0 h} u\right)+\left[\tilde{A}\left(\Psi_{h}, \Phi_{h}\right)-A_{h}^{N I}\left(\Psi_{h}, \Phi_{h}\right)\right] \\
&-\left[\tilde{A}\left(\Psi, \Phi_{h}\right)+\tilde{b}\left(\Phi_{h}, u\right)\right] .
\end{aligned}
$$


Applying the triangular inequality and the continuity of the bilinear forms $\tilde{A}(\cdot, \cdot)$ and $\tilde{b}(\cdot, \cdot)$ in $(4.94)$, we get from (4.93):

$$
\begin{aligned}
\left\|u_{h}-\mathcal{P}_{0 h} u\right\|_{1, \Omega_{h}} \leq & 1 / \beta\left\{\left[\tilde{M}\left\|\Psi-\Psi_{h}\right\|_{0, \Omega_{h}}+\tilde{m}\left\|u-\mathcal{P}_{0 h} u\right\|_{1, \Omega_{h}}\right]+\left[\sup _{\Phi_{h} \in \mathbf{V}_{h}-\{0\}} \frac{\left|\tilde{A}\left(\Psi, \Phi_{h}\right)+\tilde{b}\left(\Phi_{h}, u\right)\right|}{\left\|\Phi_{h}\right\|_{1, \Omega_{h}}}\right]\right. \\
& \left.+\left[\sup _{\Phi_{h} \in \mathbf{V}_{h}-\{0\}} \frac{\left|\tilde{A}\left(\Psi_{h}, \Phi_{h}\right)-A_{h}^{N I}\left(\Psi_{h}, \Phi_{h}\right)\right|}{\left\|\Phi_{h}\right\|_{1, \Omega_{h}}}\right]\right\}
\end{aligned}
$$

Then, applying (4.92) and Propositions 4.3 and 4.4, we have

$$
\left\|u_{h}-\mathcal{P}_{0 h} u\right\|_{1, \Omega_{h}} \leq C\left[h^{1 / 2}\left(\|u\|_{3, \Omega}+\sqrt{h}\|\Psi\|_{1, \Omega}\right)+h^{3 / 2}\|u\|_{3, \Omega}+h\|A\|_{1, \infty, \Omega}\left\|\Psi_{h}\right\|_{0, \Omega_{h}} h^{3 / 2}(1+\sqrt{h})\|u\|_{3, \Omega}\right] .
$$

But

$$
\begin{aligned}
\left\|\Psi_{h}\right\|_{0, \Omega_{h}} & \leq\left\|\Psi-\Psi_{h}\right\|_{0, \Omega_{h}}+\|\Psi\|_{0, \Omega_{h}} \leq C h^{1 / 2}\left(\|u\|_{3, \Omega}+\sqrt{h}\|\Psi\|_{1, \Omega}\right)+\|\Psi\|_{0, \Omega} \\
& \leq C\left(\|u\|_{3, \Omega}+\|\Psi\|_{1, \Omega}\right) .
\end{aligned}
$$

From (4.96) and (4.97),

$$
\begin{aligned}
\left\|u_{h}-\mathcal{P}_{0 h} u\right\|_{1, \Omega_{h}} & \leq C\left[h^{1 / 2}\left(\|u\|_{3, \Omega}+h^{1 / 2}\|\Psi\|_{1, \Omega}\right)+\left(h^{3 / 2}+h+h \sqrt{h}(1+\sqrt{h})\right)\|u\|_{3, \Omega}+h\|\Psi\|_{1, \Omega}\right] \\
& \leq C h^{1 / 2}\left[\|u\|_{3, \Omega}+h^{1 / 2}\|\Psi\|_{1, \Omega}\right] .
\end{aligned}
$$

Then,

$$
\begin{aligned}
\left\|u-u_{h}\right\|_{1, \Omega_{h}} & \leq\left\|u-\mathcal{P}_{0 h} u\right\|_{1, \Omega_{h}}+\left\|\mathcal{P}_{0 h} u-u_{h}\right\|_{1, \Omega_{h}} \leq C\left(h^{3 / 2}\|u\|_{3, \Omega}+h^{1 / 2}\|u\|_{3, \Omega}+h\|\Psi\|_{1, \Omega}\right) \\
\Longrightarrow\left\|u-u_{h}\right\|_{1, \Omega_{h}} & \leq C h^{1 / 2}\left(\|u\|_{3, \Omega}+h^{1 / 2}\|\Psi\|_{1, \Omega}\right)
\end{aligned}
$$

with $C>0$ independent of $h$.

Remark 4.2. Error estimate (4.79) [resp. (4.80)] depends on the estimates of the three terms occurring due to the errors involved with

(i) interpolation;

(ii) approximation of the curved boundary $\Gamma$ by the polygon $\Gamma_{h}$;

(iii) non-exact integration

i.e. the terms in the second and third square brackets on the right-hand side of (4.87) (resp. (4.95)) correspond to (ii) and (iii) respectively, and the terms in the first square bracket correspond to (i) and also indirectly to (ii) [see (4.6)]. Hence, it will be interesting to study the two particular cases:

Case 1: there is no approximation of boundary, in other words $\Gamma$ is a polygon, but numerical integration is performed, i.e. error due to (iii) is present, but an error due to (ii) is absent;

Case 2: polygonal boundary approximation is made,but no numerical integration is necessary and hence, it is not performed i.e. error due to (ii) is present, but an error due to (iii) is absent. 
Case 1. $\Gamma$ is a (straight) polygonal boundary of the convex polygonal domain $\Omega$ which is considered in all papers $[4,5,15,28,33]$ etc., i.e.

$$
\Gamma=\Gamma_{h}, \Omega=\Omega_{h}, \bar{\Omega}=\bar{\Omega}_{h}=\cup_{T \in \tau_{h}} T \forall h>0
$$

$\Longrightarrow$ error due to (ii) is absent. Moreover, using higher order elements i.e. $P_{m}$-elements with $m>2$, to construct finite element spaces, a remarkable improvement in the error estimates, i.e. $\left\|\Psi-\Psi_{h}\right\|_{0, \Omega}=O\left(h^{m-1}\right)$, $\left\|u-u_{h}\right\|_{1, \Omega}=O\left(h^{m-1}\right), m \geq 2$ can be obtained under some additional assumptions on the regularity of solution and the use of quadrature schemes with higher degree of accuracy. In fact, (4.100) holds, and $P_{m}$-elements with $m \geq 2$ can be used to define $X_{h} \subset H^{1}(\Omega), \mathbf{V}_{h} \subset \mathbf{V}, W_{h} \subset H_{0}^{1}(\Omega)$, i.e.

$$
\begin{aligned}
& X_{h}=\left\{\chi_{h}: \chi_{h} \in C^{0}(\bar{\Omega}), \chi_{h} \downarrow_{T} \in P_{m}(T) \quad \forall T \in \tau_{h}\right\} \subset H^{1}(\Omega), \\
& \mathbf{V}_{h}=\left\{\Phi_{h}: \Phi_{h}=\left(\phi_{h i j}\right)_{1 \leq i, j \leq 2} \text { with } \phi_{h i j}=\phi_{h j i} \in X_{h}\right\} \subset \mathbf{V}, \\
& W_{h}=\left\{\chi_{h}: \chi_{h} \in X_{h}, \chi_{h} \downarrow_{\Gamma}=0\right\} \subset H_{0}^{1}(\Omega) .
\end{aligned}
$$

Then, we use quadrature schemes (3.11) with higher degrees of accuracy:

(A3) $A_{h}^{N I}(\cdot, \cdot)\left(\right.$ resp. $\left.b_{h}^{N I}(\cdot, \cdot)\right)$ defined by $(3.23)$ [resp. (3.24)] corresponds to the quadrature scheme (3.11) with $i=1$ [resp. $i=2]$ which is exact for $P_{3 m-2}(\hat{T})$ [resp. $\left.P_{2 m-2}(\hat{T})\right]$.

Following the steps of the proofs of (3.27) and (3.28), we have: $\exists \alpha_{0}>0$, independent of $h$ such that

$$
A_{h}^{N I}\left(\Phi_{h}, \Phi_{h}\right) \geq \alpha_{0}\left\|\Phi_{h}\right\|_{0, \Omega}^{2} \quad \forall \Phi_{h} \in \mathbf{V}_{h} \subset \mathbf{V}
$$

$\exists \beta_{1}>0$, independent of $h$ such that

$$
\sup _{\Phi_{h} \in \mathbf{V}_{h}-\{0\}} \frac{\left|b_{h}^{N I}\left(\Phi_{h}, \chi_{h}\right)\right|}{\left\|\Phi_{h}\right\|_{\mathbf{V}}} \geq \beta_{1}\left\|\chi_{h}\right\|_{1, \Omega} \quad \forall \chi_{h} \in W_{h} \subset H_{0}^{1}(\Omega)
$$

and the corresponding $\left(\mathbf{Q}_{\mathbf{h}}\right)$ has a unique solution $\left(\Psi_{h}, u_{h}\right) \in \mathbf{V}_{h} \times W_{h}$.

Moreover,

$$
(4.100) \Longrightarrow \tilde{A}(\cdot, \cdot)=A(\cdot, \cdot), \tilde{b}(\cdot, \cdot)=b(\cdot, \cdot)
$$

and

$$
\left|\tilde{A}\left(\Psi, \Phi_{h}\right)+\tilde{b}\left(\Phi_{h}, u\right)\right|=\left|A\left(\Psi, \Phi_{h}\right)+b\left(\Phi_{h}, u\right)\right|=0 \quad \forall \Phi_{h} \in \mathbf{V}_{h} \subset \mathbf{V}
$$

in Proposition 4.4 by virtue of the first equation (2.10);

$$
\left|b\left(\Phi_{h}, \chi_{h}\right)-b_{h}^{N I}\left(\Phi_{h}, \chi_{h}\right)\right|=0 \quad \forall \Phi_{h} \in \mathbf{V}_{h}, \forall \chi_{h} \in W_{h}
$$

with $\mathbf{V}_{h}$ and $W_{h}$ defined by (4.101), since the quadrature scheme used in $b_{h}^{N I}(\cdot, \cdot)$ is exact for $P_{2 m-2}(\hat{T}), m \geq 2$. Proposition 4.3 is replaced by the following result, whose proof is analogous.

Under (A3), for $A_{i j k l} \in W^{m-1, \infty}(\Omega)$ with $m \geq 2 \forall i, j, k, l=1,2$, we have

$$
\left|A\left(\Phi_{h}, \underline{\sigma}_{h}\right)-A_{h}^{N I}\left(\Phi_{h}, \underline{\sigma}_{h}\right)\right| \leq C h^{m-1}\left\|\Phi_{h}\right\|_{0, \Omega}\left\|_{h}\right\|_{0, \Omega} \quad \forall \Phi_{h}, \underline{\sigma}_{h} \in \mathbf{V}_{h} \subset \mathbf{V} .
$$

By virtue of (4.100), the interpolation operators $\mathcal{P}_{h}$ and $\mathcal{P}_{0 h}$ defined in (4.1) and (4.4) respectively are now identical, i.e.

$$
\mathcal{P}_{h} \chi=\mathcal{P}_{0 h} \chi \in W_{h} \subset H_{0}^{1}(\Omega) \quad \forall \chi \in H^{s}(\Omega) \cap H_{0}^{1}(\Omega) \quad \text { with } s \geq 2,
$$


and estimates (4.5) and (4.6) are replaced by the classical estimates:

$$
\forall \chi \in H^{s}(\Omega) \cap H_{0}^{1}(\Omega) \text { with } s \geq 2, \quad\left\|\chi-\mathcal{P}_{0 h} \chi\right\|_{r, \Omega} \leq C h^{s-r}|\chi|_{s, \Omega} \quad(s \geq 2 ; r=0,1) .
$$

Lemma 4.2 holds with $b_{h}^{N I}(\cdot, \cdot)$ corresponding to the quadrature scheme (3.11) with $i=2$, which is exact for $P_{2 m-2}(\hat{T})$. Then, $\forall \Phi=\left(\phi_{i j}\right)_{1 \leq i, j \leq 2}$ with $\phi_{i j}=\phi_{j i} \in H^{m-1}(\Omega), m \geq 2, \exists \Theta_{h} \in \mathbf{V}_{h} \subset \mathbf{V}$ satisfying $b\left(\Phi, \chi_{h}\right)=b_{h}^{N I}\left(\Theta_{h}, \chi_{h}\right) \forall \chi_{h} \in W_{h} \subset H_{0}^{1}(\Omega)$ such that

$$
\left\|\Phi-\Theta_{h}\right\|_{r, \Omega} \leq C h^{m-r-1}\|\Phi\|_{m-1, \Omega}(r=0,1) .
$$

Now, following the steps of the proof of Theorem 4.1, using assumption (A3) and (4.100-4.110), assuming that $u \in H^{m+1}(\Omega) \cap H_{0}^{2}(\Omega)$ with $m \geq 2$ is the unique solution of $\left(\mathbf{P}_{\mathbf{G}}\right)$ in $(2.3-2.4)$ and $(\Psi, u)$ is the unique solution of $(\mathbf{Q})$ with $\Psi=\left(\psi_{i j}\right)_{1 \leq i, j \leq 2}, \quad \psi_{i j}=\psi_{j i} \in H^{m-1}(\Omega) \forall i, j=1,2$,

$$
\begin{aligned}
\left\|\Psi-\Psi_{h}\right\|_{0, \Omega} & \leq C h^{m-1}\left[\|u\|_{m+1, \Omega}+\|\Psi\|_{m-1, \Omega}\right], \\
\left\|u-u_{h}\right\|_{1, \Omega} & \leq C h^{m-1}\left[\|u\|_{m+1, \Omega}+\|\Psi\|_{m-1, \Omega}\right] .
\end{aligned}
$$

Estimates (4.111) and (4.112) are of the same order $O\left(h^{m-1}\right), m \geq 2$ as obtained in [5] (resp. for H-H-M mixed scheme for the biharmonic problem (2.20-2.21) by Brezzi-Raviart in [15], pages 16-17) under the same regularity assumptions, when errors due to (ii) and (iii) are absent, i.e. when $\Gamma$ is a polygon and exact integration is performed.

For $m=1$, neither the estimates of [5] nor those of Brezzi-Raviart in [15] hold (see Remark 2 of [15], page 20), but Miyoshi obtained estimates of order $O\left(h^{1 / 2}\right)$ for $m=1$ in [28], in which the elegant, systematic mixed method analysis of Babuška-Brezzi-Raviart has not been followed (see also [34])!

Hence, based on Babuška-Brezzi-Raviart mixed method analysis, best available error estimates for this problem using $P_{2}$ elements are of order $O(h)$ [5], when errors due to (ii) and (iii) are absent. Moreover, when quadrature schemes with higher degrees of accuracy $P_{3 m-2}(\hat{T})$ for $A_{h}^{N I}(\cdot, \cdot)$ and $P_{2 m-2}(\hat{T})$ for $b_{h}^{N I}(\cdot, \cdot)(m \geq 2)$ are used, the error due to only (iii) is of the order $O\left(h^{m-1}\right)$ [see (4.107)].

Case 2. Curved boundary $\Gamma$ is approximated by a polygon $\Gamma_{h}$ as in (3.1-3.6), but exact integration is possible and performed i.e. only error due to (ii) is present. Since exact integration is performed, $\tilde{A}(\cdot, \cdot)=$ $A_{h}^{N I}(\cdot, \cdot), \tilde{b}(\cdot, \cdot)=b_{h}^{N I}(\cdot, \cdot)$ and the term in the third square bracket on the right-hand side of (4.87) [resp. (4.95)] vanishes.

For polygonal approximation $\Gamma_{h}$ to $\Gamma$, we have meas $\left(\omega_{h}\right)=O\left(h^{2}\right)$ with $\omega_{h}=\Omega-\Omega_{h}$. Consequently, from $(4.42,4.44,4.45)$ in the proof of Proposition 4.4,

$$
\left|\tilde{A}\left(\Psi, \Psi_{h}-\Phi_{h}\right)+\tilde{b}\left(\Psi_{h}-\Phi_{h}, u\right)\right|=O\left(h^{3 / 2}\right),
$$

even if $P_{m}$-elements with $m \geq 2$ are used to define finite element spaces $\mathbf{V}_{h}$ and $W_{h}$ [see (4.101)]. In other words, by using $P_{m}$-elements with $m>2$, the estimate (4.113) can not be improved unless better approximation of $\Gamma$ is made. Incidentally, this is exactly the reason for using $P_{2}$-elements in the definition of $\mathbf{V}_{h}$ and $W_{h}$ in (3.18) and (3.19) respectively.

Again, from Remark 4.1, we find that for

$$
u \in H^{m+1}(\Omega) \cap H_{0}^{2}(\Omega) \subset H^{m+1}(\Omega) \cap H_{0}^{1}(\Omega), \quad\left\|u-\mathcal{P}_{0 h} u\right\|_{1, \Omega_{h}}=O\left(h^{3 / 2}\right) \forall m \geq 2,
$$


which can not be improved upon unless boundary approximation is improved. Hence, for polygonal boundary approximation, we find from (4.113) and (4.114) that the estimates of order $O\left(h^{3 / 2}\right)$ can not be improved upon by any choice of $m>2$

(i) in the definition of $\mathbf{V}_{h}$ and $W_{h}$ in (4.101) and

(ii) in the regularity of solution $u \in H^{m+1}(\Omega) \cap H_{0}^{2}(\Omega)$ of $\left(\mathbf{P}_{\mathbf{G}}\right)$, i.e. the optimal case is $m=2$.

Finally, the use of the inverse inequality (4.88) in (4.91) is necessary (see $[5,14,15,18]$ ) and gives the estimate:

$$
\left\|\Psi-\Psi_{h}\right\|_{0, \Omega_{h}}=O\left(h^{1 / 2}\right),
$$

which is used to get the estimate:

$$
\left\|u-u_{h}\right\|_{1, \Omega_{h}}=O\left(h^{1 / 2}\right) .
$$

Thus, for this crude but most important and commonly used polygonal approximation $\Gamma_{h}$ to $\Gamma$, there is a loss in the exponent of $h$ by only ' $\mathbf{1} / \mathbf{2}$ ' in the estimates (4.115-4.116), the best available estimates [5], [15] based on Babuška-Brezzi-Raviart mixed method analysis being $\left\|\Psi-\Psi_{h}\right\|_{0, \Omega}=O(h), \quad\left\|u-u_{h}\right\|_{1, \Omega}=O(h)$ for $m=2$, when there is neither boundary approximation nor non-exact integration (see also Case 1 above for $m=2)$. In fact, in [9], the estimates $\left\|\Psi-\Psi_{h}\right\|_{0, \Omega_{h}}=O(h),\left\|u-u_{h}\right\|_{1, \Omega_{h}}=O(h)$ have been obtained when $\Gamma$ has been approximated by a curved boundary $\Gamma_{h}$ constructed with the help of isoparametric mapping, for which $\bar{\Omega}_{h} \not \subset \bar{\Omega}, \quad \bar{\Omega} \not \subset \bar{\Omega}_{h}$ and $\bar{\Omega}_{h}$ is no longer convex in general. Consequently, a completely different, independent analysis has been developed in [9].

Hence, it is obvious from the facts explained above that for polygonal approximation $\Gamma_{h}$, the estimates $\left\|\Psi-\Psi_{h}\right\|_{0, \Omega_{h}}=O\left(h^{1 / 2}\right)$ and $\left\|u-u_{h}\right\|_{1, \Omega_{h}}=O\left(h^{1 / 2}\right)$ are the 'best' ones based on Babuška-Brezzi-Raviart mixed method analysis for fourth order problems.

The authors express their special thanks to the referee for all his suggestions for the improvement of the paper and particularly for pointing out the necessity of substantiation of the interpolation estimates in (4.5-4.6).

\section{REFERENCES}

[1] R.A. Adams, Sobolev Spaces. Academic Press, NewYork (1975).

[2] I. Babuška, Error Bounds for Finite Element Method. Numer. Math. 16 (1971) 322-333.

[3] I. Babuška and J.E. Osborn, Eigenvalue Problems, in Handbook of Numerical Analysis, Vol. 2, Part 1, J.L. Lions and P.G. Ciarlet Eds., North-Holland, Amsterdam (1991) 641-787.

[4] S. Balasundaram and P.K. Bhattacharyya, On Existence of Solution of the Dirichlet Problem of Fourth Order Partial Differential Equations with Variable Coefficients. Quart. Appl. Math. 39 (1983) 311-317.

[5] S. Balasundaram and P.K. Bhattacharyya, A Mixed Finite Element Method for Fourth Order Elliptic Equations with Variable Coefficients. Comput. Math. Appl. 10 (1984) 245-256.

[6] S. Balasundaram and P.K. Bhattacharyya, A Mixed Finite Element Method for Fourth Order Elliptic Operators with Variable Coefficients, 4th Int. Symp. on Finite Element Methods in Flow Problems, Chuo University, Tokyo (1982), in Finite Element Analysis of Flow Problems, T. Kawai Ed., Tokyo University Press (1982) 995-1001.

[7] S. Balasundaram and P.K. Bhattacharyya, A Mixed Finite Element Method for Fourth Order Partial Differential Equations. ZAMM 66 (1986) 489-499.

[8] M. Bernadou, Méthodes d'éléments finis pour les problèmes de coques minces. Collection Recherches en Mathematiques Appliquees, Masson, Paris (1994).

[9] P.K. Bhattacharyya and N. Nataraj, Error Estimates for Isoparametric Mixed Finite Element Solution of 4th Order Elliptic Problems with Variable Coefficients (submitted).

[10] P.K. Bhattacharyya, A Mixed Finite Element Method for Fourth Order Elliptic Operators with Variable Coefficients, in Proc. of 6th Joint France-Italy-USSR Symp. on Numerical Solution of Nonlinear Problems, INRIA, France (1983) 127-144; Russian Translation, in Methods of Computational Mathematics and Mathematical Modelling, Computational Mathematics Section, Academy of Sciences, Moscow, USSR (1985) 76-99. 
[11] P.K. Bhattacharyya, S. Gopalsamy and S. Balasundaram, On a Mixed Finite Element Method for Clamped Anisotropic Plate Bending Problems. Internat. J. Numer. Methods Engrg. 28 (1989) 1351-1370.

[12] P.K. Bhattacharyya and S. Balasundaram, A Mixed Finite Element Method for Fourth Order Elliptic Problems with Variable Coefficients. J. Comput. Appl. Math. 22 (1988) 1-24.

[13] F. Brezzi, On the Existence, Uniqueness and Approximation of Saddle-Point Problems Arising from Lagrangian Multipliers. RAIRO Anal. Numér. 8 (1974) 129-151.

[14] F. Brezzi and M. Fortin, Mixed and Hybrid Finite Element Methods. Springer-Verlag (1991).

[15] F. Brezzi and P.A. Raviart, Mixed Finite Element Methods for 4th Order Elliptic Equations, in Topics in Numerical Analysis III, J. Miller Ed., Academic Press, New York (1978) 33-56.

[16] P.G. Ciarlet, Basic Error Estimates for Elliptic Problems, in Handbook of Numerical Analysis, Vol. 2, Part 1, J.L. Lions and P.G. Ciarlet Eds., North-Holland, Amsterdam (1991).

[17] P.G. Ciarlet, The Finite Element Method for Elliptic Problems. North-Holland, Amsterdam (1978).

[18] P.G. Ciarlet and P.A. Raviart, A Mixed Finite Element Method for the Biharmonic Equation, in Symp. on Mathematical Aspects of Finite Elements in Partial Differential Equations, C. de Boor Ed., Academic Press, New York (1974) $125-145$.

[19] P.G. Ciarlet and P.A Raviart, The Combined Effect of Curved Boundaries and Numerical Integration in Isoparametric Finite Element Methods, in The Mathematical Foundations of the Finite Element Method with Applications to Partial Differential Equations, A.K. Aziz Ed., Academic Press, New York (1972) 409-474.

[20] S. Gopalsamy and P.K. Bhattacharyya, On Existence and Uniqueness of Solution of Boundary Value Problems of Fourth Order Elliptic Partial Differential Equations with Variable Coefficients. J. Math. Anal. Appl. 136 (1988) 589-608.

[21] P. Grisvard, Elliptic Problems in Nonsmooth Domains. Pitman, Boston (1985).

[22] K. Hellan, Analysis of Elastic Plates in Flexure by a Simplified Finite Element Method. Acta Polytech. Scand., Civil Engineering Series, Trondheim, 46 (1967).

[23] L. Herrmann, Finite Element Bending Analysis for Plates. J. Eng. Mech. Div. ASCE 93, EM 5 (1967) 49-83.

[24] V.A. Kondratev, Boundary Value Problems for Elliptic Equations in Domains with Conical or Angular Points. Trudy Moskov. Mat. Obshch. 16 (1967) 7209-292.

[25] S.G. Leknitskii, Anisotropic Plates. Gordon and Breach Science Publishers, New York (1968).

[26] J.L. Lions, Problèmes aux Limites dans les Équations aux Derivées Partielles. Les Presses de l’Université de Montréal, Montréal (1965).

[27] L. Mansfield, Approximation of the Boundary in the Finite Element solution of fourth order problems. SIAM J. Numer. Anal. 15 (1978) 568-579.

[28] T. Miyoshi, A finite element method for the solution of fourth order partial differential equations. Kumamoto J. Sci. (Math.) 9 (1973) $87-116$.

[29] N. Nataraj, P.K. Bhattacharyya, S. Balasundaram and S. Gopalsamy, On a Mixed-Hybrid Finite Element Method for Anisotropic Plate Bending Problems. Internat. J. Numer. Methods Engrg. 39 (1996) 4063-4089.

[30] J.T. Oden and G.F. Carey, Finite Elements-Mathematical Aspects IV, The Texas Finite Element Series. Prentice Hall, Eaglewood Cliffs, New Jersey (1983).

[31] A.B. Ouaritini, Méthodes d'éléments finis mixtes pour des problèmes de coques minces. Ph.D. thesis, University of Pau et Pays de L'Adour, France (1984).

[32] P.A. Raviart and J.M. Thomas, Introduction à l'Analyse Numérique des Équations aux Dérivées Partielles. Mason, Paris (1983).

[33] J.E. Roberts and J.M. Thomas, Mixed and Hybrid Methods, in Handbook of Numerical Analysis, Vol. 2, Part 1, J.L Lions and P.G. Ciarlet Eds., North-Holland, Amsterdam (1991) 523-633.

[34] R. Scholtz, A Mixed Method for 4th order Problems Using Linear Finite Elements. RAIRO Anal. Numér. 12 (1978) 85-90.

[35] R. Scott, Finite Element Techniques for Curved Boundaries. Ph.D. thesis, M.I.T. (1973).

[36] G. Strang and G.J. Fix, An Analysis of the Finite Element Method. Prentice-Hall, New York (1973).

[37] S. Timoshenko and S. Woinowsky-Kreiger, Theory of Plates and Shells. McGraw-Hill Book Company, New York (1959).

[38] A. Zeníšek, Nonlinear Elliptic and Evolution Problems and their Finite Element Approximations. Academic Press, New York (1990).

[39] M. Zlamal, Curved elements in the finite element method, I. SIAM J. Numer. Anal. 10 (1973) 229-240.

[40] M. Zlamal, Curved elements in the finite element method, II. SIAM J. Numer. Anal. 11 (1974) 347-362. 